\title{
Scratchpad Sharing in GPUs
}

\author{
VISHWESH JATALA, Indian Institute of Technology, Kanpur \\ JAYVANT ANANTPUR, Indian Institute of Science, Bangalore \\ AMEY KARKARE, Indian Institute of Technology, Kanpur
}

General-Purpose Graphics Processing Unit (GPGPU) applications exploit on-chip scratchpad memory available in the Graphics Processing Units (GPUs) to improve performance. The amount of thread level parallelism (TLP) present in the GPU is limited by the number of resident threads, which in turn depends on the availability of scratchpad memory in its streaming multiprocessor (SM). Since the scratchpad memory is allocated at thread block granularity, part of the memory may remain unutilized. In this article, we propose architectural and compiler optimizations to improve the scratchpad memory utilization. Our approach, called Scratchpad Sharing, addresses scratchpad under-utilization by launching additional thread blocks in each SM. These thread blocks use unutilized scratchpad memory and also share scratchpad memory with other resident blocks. To improve the performance of scratchpad sharing, we propose Owner Warp First $(O W F)$ scheduling that schedules warps from the additional thread blocks effectively. The performance of this approach, however, is limited by the availability of the part of scratchpad memory that is shared among thread blocks.

We propose compiler optimizations to improve the availability of shared scratchpad memory. We describe an allocation scheme that helps in allocating scratchpad variables such that shared scratchpad is accessed for short duration. We introduce a new hardware instruction, relssp, that when executed releases the shared scratchpad memory. Finally, we describe an analysis for optimal placement of relssp instructions, such that shared scratchpad memory is released as early as possible, but only after its last use, along every execution path.

We implemented the hardware changes required for scratchpad sharing and the relssp instruction using the GPGPU-Sim simulator and implemented the compiler optimizations in Ocelot framework. We evaluated the effectiveness of our approach on 19 kernels from 3 benchmarks suites: CUDA-SDK, GPGPUSim, and Rodinia. The kernels that under-utilize scratchpad memory show an average improvement of $19 \%$ and maximum improvement of $92.17 \%$ in terms of the number of instruction executed per cycle when compared to the baseline approach, without affecting the performance of the kernels that are not limited by scratchpad memory.

CCS Concepts: $\bullet$ Computer systems organization $\rightarrow$ Single instruction, multiple data; $\bullet$ Software and its engineering $\rightarrow$ Compilers;

Additional Key Words and Phrases: Scratchpad sharing, thread level parallelism, control flow graph, code motion

Vishwesh Jatala is supported by TCS Ph.D. fellowship.

Extension of Conference Paper: "Improving GPU Performance Through Resource Sharing", in HPDC 2016 [Jatala et al. 2016a]. We extend the previous work by introducing compiler optimizations to leverage the resource sharing approach.

Authors' addresses: V. Jatala, Department of CSE, Indian Institute of Technology, Kanpur, Uttar Pradesh 208016, India; email: vjatala@cse.iitk.ac.in; J. Anantpur, Supercomputer Education and Research Centre (SERC), Indian Institute of Science, Bangalore, Karnataka 560012, India; email: jayvant.anantpur@ gmail.com; A. Karkare, Department of CSE, Indian Institute of Technology, Kanpur, Uttar Pradesh 208016, India; email: karkare@cse.iitk.ac.in.

Permission to make digital or hard copies of part or all of this work for personal or classroom use is granted without fee provided that copies are not made or distributed for profit or commercial advantage and that copies show this notice on the first page or initial screen of a display along with the full citation. Copyrights for components of this work owned by others than ACM must be honored. Abstracting with credit is permitted. To copy otherwise, to republish, to post on servers, to redistribute to lists, or to use any component of this work in other works requires prior specific permission and/or a fee. Permissions may be requested from Publications Dept., ACM, Inc., 2 Penn Plaza, Suite 701, New York, NY 10121-0701 USA, fax +1 (212) 869-0481, or permissions@acm.org.

(c) 2017 ACM 1544-3566/2017/05-ART15 $\$ 15.00$

DOI: http://dx.doi.org/10.1145/3075619 
ACM Reference Format:

Vishwesh Jatala, Jayvant Anantpur, and Amey Karkare. 2017. Scratchpad sharing in GPUs. ACM Trans. Archit. Code Optim. 14, 2, Article 15 (May 2017), 29 pages.

DOI: http://dx.doi.org/10.1145/3075619

\section{INTRODUCTION}

The throughput achieved by a Graphics Processing Unit (GPU) depends on the amount of thread-level-parallelism (TLP) it utilizes. Therefore, improving the TLP of GPUs has been the focus of many recent studies [Kayiran et al. 2013; Anantpur and Govindarajan 2014; Xie et al. 2015; Fung et al. 2007]. The TLP present in a GPU is dependent on the number of resident threads. A programmer interested in parallelizing an application in GPU invokes a function, called kernel, with a configuration consisting of number of thread blocks and number of threads in each thread block. The maximum number of thread blocks, and hence the number of threads, that can be launched in a Streaming Multiprocessor (SM) depends on the number of available resources (such as scratchpad memory $^{1}$ and registers) in it. If an SM has $\mathrm{R}$ resources and each thread block requires $R_{t b}$ resources, then $\left\lfloor R / R_{t b}\right\rfloor$ number of thread blocks can be launched in each SM. Thus, utilizing $R_{t b} *\left\lfloor R / R_{t b}\right\rfloor$ units of resources present in the SM, the remaining $R$ mod $R_{t b}$ resources are wasted.

This underutilization of resources is evident from Figures 1 and 2. For the benchmark applications in Table I and for the GPU configuration in Table II, Figure 1 shows the number of thread blocks that are launched in each SM, and Figure 2 shows the percentage of unutilized scratchpad memory.

Example 1.1. Consider the application backprop in Table I. It requires 9408 bytes of scratchpad memory to launch a thread block in the SM. According to the GPU configuration shown in Table II, each SM has 16K bytes of scratchpad memory. Hence, only one thread block can be launched in the SM, this utilizes 9,408 bytes of scratchpad memory. The remaining 6,976 bytes of scratchpad memory remains unutilized. We can observe the similar behavior for other applications as well. Hence, scratchpad allocation at thread block level granularity not only has a lower number of resident thread blocks but also has scratchpad memory underutilization.

In this article, we propose an approach called Scratchpad Sharing, which launches additional thread blocks in each SM. These thread blocks help in improving the TLP by utilizing the wasted scratchpad memory and by sharing the scratchpad memory with the other resident thread blocks. However, it is observed that increasing the number of thread blocks can also lead to a decrease in the throughput due to resource contention [Kayiran et al. 2013]. To overcome this, we propose Owner Warp First (OWF), a warp scheduling algorithm that improves performance by effectively scheduling warps to minimize the dependency for shared scratchpad memory.

In our experiments, we observed that the performance of scratchpad sharing depends on the availability of the scratchpad memory that is shared between the thread blocks. We have developed static analysis that helps in allocating scratchpad variables into shared and unshared scratchpad regions, such that the shared scratchpad variables are needed only for a short duration. We modified the GPU architecture to include a new hardware instruction (relssp) to release the acquired shared scratchpad memory at run-time. When all the threads of a thread block execute the relssp instruction, the thread block releases its shared scratchpad memory. We describe an algorithm to help

\footnotetext{
${ }^{1}$ While the term shared memory is more popular, we use scratchpad memory in the article to avoid using "shared shared memory" for the part of the shared memory that is shared among thread blocks in our approach.
} 
Table I. Benchmark Applications for which the Number of Thread Blocks is Limited by Scratchpad Memory

\begin{tabular}{|c|c|c|c|c|c|c|}
\hline \multicolumn{2}{|r|}{ Benchmark } & Application & Kernel & $\begin{array}{c}\text { \#Scratchpad } \\
\text { Variables }\end{array}$ & $\begin{array}{c}\text { Scratchpad } \\
\text { Size (Bytes) }\end{array}$ & $\begin{array}{c}\text { Block } \\
\text { Size }\end{array}$ \\
\hline \multicolumn{7}{|c|}{ Set-1: Shared scratchpad can be released before the end of the kernel } \\
\hline 1. & RODINIA & backprop & bpnn_layerforward_CUDA & 2 & 9408 & 256 \\
\hline 2. & CUDA-SDK & det8x8_1 (DCT1) & CUDAkernel2DCT & 1 & 2112 & 64 \\
\hline 3. & CUDA-SDK & det8x8_2 (DCT2) & CUDAkernel2IDCT & 1 & 2112 & 64 \\
\hline 4. & CUDA-SDK & dct8x8_3 (DCT3) & CUDAkernelShortDCT & 1 & 2176 & 128 \\
\hline 5. & CUDA-SDK & det8x8_4 (DCT4) & CUDAkernelShortIDCT & 1 & 2176 & 128 \\
\hline 6. & GPGPU-SIM & $\mathrm{NQU}$ & solve_nqueen_cuda_kernel & 5 & 10496 & 64 \\
\hline 7. & RODINIA & srad_v2_1 (SRAD1) & srad_cuda_1 & 6 & 13824 & 576 \\
\hline 8. & RODINIA & srad_v2_2 (SRAD2) & srad_cuda_2 & 5 & 11520 & 576 \\
\hline \multicolumn{7}{|c|}{ Set-2: Shared scratchpad can not be released before the end of the kernel } \\
\hline & CUDA-SDK & FDTD3d & FiniteDifferencesKernel & 1 & 3840 & 128 \\
\hline 10. & RODINIA & heartwall & kernel & 8 & 11872 & 128 \\
\hline 11. & CUDA-SDK & histogram & histogram256Kernel & 1 & 9216 & 192 \\
\hline 12. & CUDA-SDK & marchingCubes (MC & generateTriangles & 2 & 9216 & 32 \\
\hline 13. & RODINIA & NW1 & needle_cuda_shared_1 & 2 & 8452 & 32 \\
\hline 14. & RODINIA & NW2 & needle_cuda_shared_2 & 2 & 8452 & 32 \\
\hline
\end{tabular}

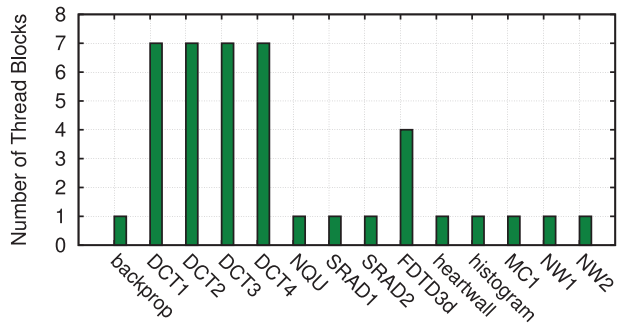

Fig. 1. Number of resident thread blocks.

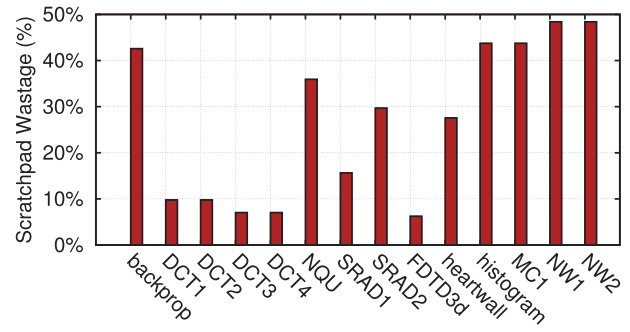

Fig. 2. Scratchpad underutilization.

compiler in an optimal placement of the relssp instruction in a kernel, such that the shared scratchpad can be released as early as possible, without causing any conflicts among shared thread blocks. These optimizations improve the availability of shared scratchpad memory.

The main contributions of this article are:

(1) We describe an approach to launch more thread blocks by sharing the scratchpad memory. We further describe a warp-scheduling algorithm that improves the performance of the GPU applications by effectively using warps from additional thread blocks.

(2) We present a static analysis to layout scratchpad variables in order to minimize the shared scratchpad region. We introduce a hardware instruction, relssp, and an algorithm for optimal placement of relssp in the user code to release the shared scratchpad region at the earliest.

(3) We used the GPGPU-Sim [Bakhoda et al. 2009] simulator and the Ocelot [Diamos et al. 2010] compiler framework to implement and evaluate our ideas. On several kernels from various benchmark suites, we achieved an average improvement of $19 \%$ and a maximum improvement of $92.17 \%$ over the baseline approach.

Section 2 describes the background required for our approach. Section 3 presents the details of the scratchpad sharing. Section 4 presents the need for compiler 


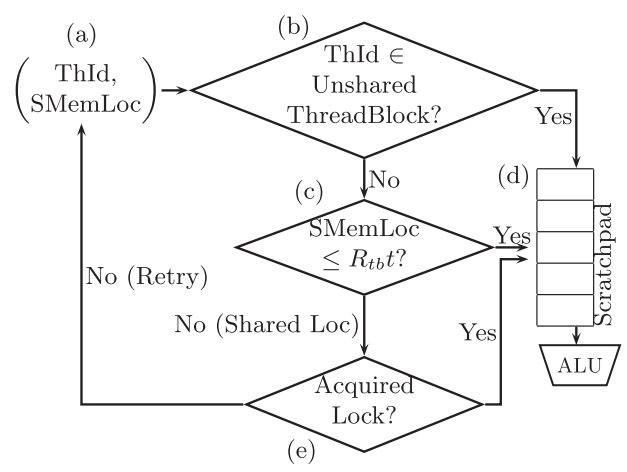

Fig. 3. Scratchpad access mechanism.
Table II. GPGPU-Sim Architecture

\begin{tabular}{l|l}
\hline \hline Resource & Configuration \\
\hline Number of SMs & 14 \\
Shader Core Clock & $732 \mathrm{MHz}$ \\
Scratchpad Memory per SM & $16 \mathrm{~KB}$ \\
Number of Registers per SM & 65536 \\
Max Number of TBs per SM & 16 \\
Max Number of Threads per SM & 3072 \\
Warp Scheduling & LRR \\
Number of Schedulers per SM & 4 \\
L1-Cache per SM & $16 \mathrm{~KB}$ \\
L2-Cache & $1.5 \mathrm{MB}$ \\
DRAM Scheduler & FR-FCFS
\end{tabular}

optimizations. The optimizations themselves are discussed in Section 5. Section 6 analyzes the hardware requirements and the complexity of our approach. Section 7 shows the experimental results. Section 8 discusses related work, and Section 9 concludes the article.

\section{BACKGROUND}

A typical NVIDIA GPU consists of a set of streaming multiprocessors (SMs). Each SM contains execution units called stream processors. A programmer can parallelize an application on GPU using programming languages such as CUDA [2012] and OpenCL [2009]. The region of a program that is to be parallelized is specified using a function called kernel. The kernel is invoked with the configuration specifying the number of thread blocks and number of threads as $<<<\#$ ThreadBlocks, \#Threads $>>>$. The number of thread blocks that are actually launched in an SM depends on the resources available in the SM, such as the amount of scratchpad memory, the number of registers. In GPUs, each SM has its own private scratchpad memory, which can be used by thread blocks that are launched in it. In CUDA, a variable can be allocated to scratchpad memory by specifying ___shared_-_ keyword inside a kernel function.

The thread blocks that are launched in the GPUs are executed independently [CUDA 2012]. This allows the thread blocks to be scheduled in any order on to the SMs. The threads that are launched in an SM are partitioned into groups of consecutive 32 threads called warps. All the threads in a warp execute the same instruction in SIMD manner. Each SM has one or more warp schedulers, which fetch a warp from the pool of available warps based on some warp scheduling algorithm. When no warp can be issued in a cycle, the cycle is said to be a stall cycle. Our approach tries to reduce these stall cycles by launching more number of thread blocks in each SM.

\section{SCRATCHPAD SHARING}

Scratchpad sharing launches additional thread blocks in each SM to reduce scratchpad underutilization and, as a result, increases TLP on a SM. Example 3.1 illustrates how this works.

Example 3.1. The performance of backprop (discussed in Example 1.1) can be improved by launching two thread blocks, say $\mathrm{TB}_{0}$ and $\mathrm{TB}_{1}$, which share the scratchpad memory. The scratchpad sharing approach allocates total $16 \mathrm{~K}$ bytes of memory together for $\mathrm{TB}_{0}$ and $\mathrm{TB}_{1}$ as follows: 6,976 bytes of scratchpad memory is allocated to each thread block independently (unshared scratchpad), while the remaining 2,432 bytes of memory (shared scratchpad) is allocated to the thread block that requires it 
first. For example, if $\mathrm{TB}_{1}$ accesses the shared scratchpad memory first, it is allocated all of the shared portion. $\mathrm{TB}_{0}$ can continue its execution till it requires shared scratchpad memory, at which point it waits. $\mathrm{TB}_{0}$ resumes its execution once $\mathrm{TB}_{1}$ finishes or releases the shared scratchpad. Thus, $\mathrm{TB}_{0}$ hides the long memory latencies of $\mathrm{TB}_{1}$, thereby improving the run-time of the application.

To generalize our idea, consider a GPU that has $R$ units of scratchpad memory per $\mathrm{SM}$, and each thread block requires $R_{t b}$ units of scratchpad memory to complete its execution. Consider a pair $\mathrm{TB}_{0}$ and $\mathrm{TB}_{1}$ of shared thread blocks. Instead of allocating $R_{t b}$ units of memory to each of $\mathrm{TB}_{0}$ and $\mathrm{TB}_{1}$, we allocate $t \times R_{t b}(0<t<1)$ units of scratchpad memory to each of them independently. This is called unshared scratchpad. We further allocate $(1-t) \times R_{t b}$ units of scratchpad memory to the pair as shared scratchpad. Thus, a total of $(1+t) \times R_{t b}$ units of scratchpad memory is allocated for both. $\mathrm{TB}_{0}$ and $\mathrm{TB}_{1}$ can access shared scratchpad memory only after acquiring an exclusive lock, in an First-Come-First-Served (FCFS) manner, to prevent concurrent accesses. Once a shared thread block ( $\mathrm{say} \mathrm{TB}_{0}$ ) acquires the lock for shared scratchpad memory, it retains the lock till the end of its execution. The other thread block $\left(\mathrm{TB}_{1}\right)$ can continue to make progress until it requires to access shared scratchpad memory, at which point it waits until $\mathrm{TB}_{0}$ releases the shared scratchpad.

The naive scratchpad sharing mechanism, where each thread block shares scratchpad memory with another resident thread block, may not give benefit over default (unshared) approach. We also need to guarantee that in sharing approach, the number of active thread blocks (not waiting for shared scratchpad) is no less than the number of thread blocks in default approach.

Example 3.2. Consider the application DCT3 that requires 2,176 bytes of scratchpad memory per thread block. For the given GPU configuration (Table II), 7 thread blocks can be launched in default mode. With scratchpad sharing, it is possible to launch 12 thread blocks (for a certain value of $t$ ). Suppose we create six pairs of thread blocks where the blocks in each pair share scratchpad. Then, in the worst case, all 12 blocks may request access to the shared portion of scratchpad. This will cause six blocks to go in waiting, while only the remaining 6 will make progress. If the shared region is sufficiently large, then the application will perform worse with scratchpad sharing.

To make sure at least seven thread blocks make progress, our approach creates only five pairs of thread blocks that share scratchpad memory, the remaining two thread blocks are not involved in sharing. Thus, at most five blocks can be waiting during execution.

In our approach, the thread blocks that share the scratchpad memory are referred to as shared thread blocks, the rest are referred to as unshared thread blocks. The computation of number of shared and unshared thread blocks is described in detail in Jatala et al. [2016a].

To implement our approach, we modify the existing scratchpad access mechanism provided by GPGPUSIM [2014] simulator. Figure 3 shows the scratchpad access mechanism that supports scratchpad sharing. When a thread (Thread Id: ThId) needs to access a scratchpad location (SMemLoc), we need to check if it is from an unshared thread block. If it belongs to an unshared thread block, then it can access the location directly from scratchpad memory (Figure 3 Step (b)). Otherwise, we need to make another check if it accesses unshared scratchpad location (Step (c)). The thread accesses unshared scratchpad location if SMemLoc $<R_{t b} t$, because we allocate $R_{t b} t$ units of scratchpad memory to each of the shared thread blocks. Otherwise, we treat the location as shared scratchpad location. A thread can access unshared scratchpad location 


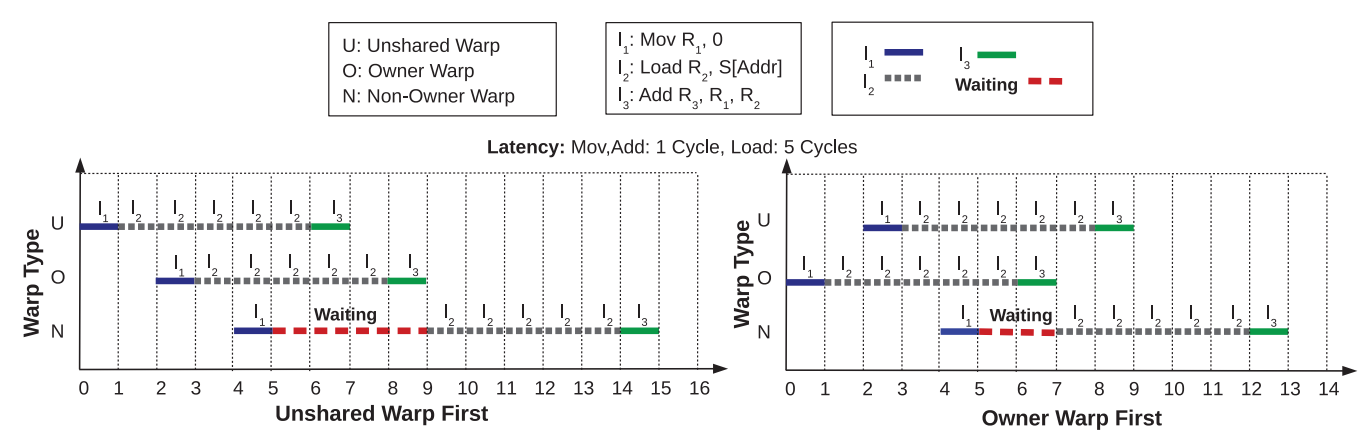

Fig. 4. Warp scheduling.

directly, however it can access the shared scratchpad location only after acquiring the exclusive lock as (Step (e)). Otherwise, it retries the access in the next cycle. ${ }^{2}$

\subsection{Owner Warp First (OWF) Scheduling}

In our approach, each SM contains various types of thread blocks such as, (1) unshared thread blocks, which do not share scratchpad memory with any other thread block, (2) shared thread blocks that own the shared scratchpad memory (Owner thread blocks) by having exclusive lock, and (3) shared thread blocks that do not own the shared scratchpad memory (Non-owner thread blocks). We refer to the warps in these thread blocks as "Unshared Warps," "Owner warps," and "Non-owner Warps," respectively. When an owner thread block finishes its execution, it transfers its ownership to its corresponding non-owner thread block, and the new thread block that will be launched becomes the non-owner thread block.

Scheduling these warps in the SM plays an important role in improving the performance of applications. Hence, we propose an optimization, Owner Warp First (OWF), that schedules the warps in the following order: (1) Owner warps, (2) Unshared warps, and (3) Non-owner warps. Giving the highest priority to owner warps helps them in finishing sooner, so the dependent non-owner warps can resume their execution, which can help in hiding long execution latencies. Figure 4 shows the benefit of giving the first priority to owner warp when compared to unshared warp. Consider an SM that has three warps: Unshared (U), Owner (O), and Non-owner (N) warps. Assume that they need to execute three instructions $I_{1}, I_{2}$, and $I_{3}$ as shown in the figure; the latency of Add and Mov instruction is 1 cycle, and the latency of Load instruction is five Cycles. Also, assume that instruction $I_{2}$ uses a shared scratchpad memory location. If unshared warp is given highest priority (shown as Unshared Warp First in the figure), then it can issue $I_{1}$ in the 1 st cycle and issue $I_{2}$ in the 2 nd cycle. However, it cannot issue $I_{3}$ in the third cycle, since $I_{3}$ is dependent on $I_{2}$ for register $R_{2}$, and $I_{2}$ takes five cycles to complete its execution. However, the owner warp with second priority can execute $I_{1}$ in the third cycle. Similarly, it can issue $I_{2}$ in the fourth cycle. The non-owner with least priority can start issuing $I_{1}$ in the fifth cycle, however, it cannot issue $I_{2}$ in the sixth cycle, since $I_{2}$ uses a shared scratchpad memory location, and it can access the shared location only after the owner thread block releases the lock, hence it waits until the owner warp finishes execution. Once the owner warp finishes the execution of $I_{2}$ and $I_{3}$ in the eighth and ninth cycles, respectively, the non-owner resumes the execution of $I_{2}$ in tenth cycle, and it can subsequently finish in 15 cycles.

\footnotetext{
${ }^{2}$ The details of required additional storage units are discussed in Section 6.
} 
If owner warp is given first priority compared to unshared warp, then it can issue $I_{1}$ and $I_{2}$ in its first and second cycles, respectively. Similarly, the unshared warp, with second priority, can issue $I_{1}$ and $I_{2}$ in third and fourth cycles. The non-owner warp with least priority can issue $I_{1}$ in fifth cycle, and it waits for owner warp to release the shared scratchpad memory. Once the owner warp completes the execution of $I_{2}$ and $I_{3}$ in seventh and eighth cycles, the non-owner can resume the execution by overlapping the execution of $I_{2}$ in the eighth cycle with unshared warp. Finally, the unshared warp and non-owner warp can finish their execution in ninth and thirteenth cycle, respectively. Thus, improving the overall performance.

\subsection{Effect of Barriers}

Our approach to scratchpad sharing does not require any special handling of barriers(_syncthreads()). Recall that in our approach, the scratchpad is shared at the thread block granularity. An owner thread block gets the lock on the shared scratchpad and releases only after it completes its execution. The warps from the non-owner thread block wait for its owner thread block for shared scratchpad memory. However, the warps from the owner thread block never wait for the warps of the non-owner thread block, since they already have lock. In the presence of _syncthreads() instruction only the warps within the same thread blocks wait for other warps, and they make progress after all the warps of the thread block arrive at the barrier [CUDA 2012]. Hence, a circular wait is not possible even in the presence of both barriers as well as shared scratchpad locks, thus avoiding deadlocks.

\subsection{Scratchpad Sharing and Thread Block Scheduling for Multiple SMs}

In the presence of multiple SMs, the additional thread blocks that are launched in each SM share scratchpad memory present in the same SM. We do not allow sharing of scratchpad memory across SMs, because access to scratchpad memory is private to each SM, and a thread block is allocated to only one SM, that is, it cannot reside partly on one SM and partly on another SM.

GPGPUSIM [2014] uses a round robin scheduling algorithm for scheduling thread blocks on multiple SMs Consider a GPU that has $p$ SMs. Assume that a kernel needs to launch $N$ thread blocks $\left(B_{1}, B_{2}, \ldots, B_{N}\right)$ in the GPU. Further, assume that default scratchpad allocation mechanism can launch $m$ thread blocks in each SM. Thus, the $i$ th SM has thread blocks with ids $B_{i}, B_{p+i}, B_{2 p+i}, \ldots B_{(m-1) p+i}$ in it. Whenever an SM finishes the execution of a thread block, a new thread block is launched in it, until all the $N$ blocks are finished. Our proposed scratchpad sharing mechanism does not modify the thread block scheduling mechanism. Assuming our approach launches $n$ blocks $(n \geq m)$, the $i$ th SM gets thread blocks with ids $B_{i}, B_{p+i}, B_{2 p+i}, \ldots, B_{(n-1) p+i}$. The additional $n-m$ thread blocks (i.e., $B_{m p+i}, B_{(m+1) p+i}, \ldots$ ) share scratchpad memory with blocks $B_{i}, B_{p+i}, \ldots$ respectively. The remaining thread blocks remain in the unsharing mode. Note that the thread blocks that share scratchpad memory are always part of the the same SM. Whenever a new thread block is launched in place of an old block that has finished its execution, the new block gets the same scratchpad sharing status (shared/unshared) as the old one.

Since thread blocks in an SM can complete their execution in any order [CUDA 2012] (no priority among thread blocks), and the scratchpad memory is shared only among the thread blocks within a SM, priority inversion problem does not arise with the scratchpad sharing approach.

\section{THE NEED FOR COMPILER OPTIMIZATIONS}

In scratchpad sharing, when two thread blocks (say, $\mathrm{TB}_{0}$ and $\mathrm{TB}_{1}$ ) are launched in shared mode, one of them accesses the shared scratchpad region at a time. As soon 


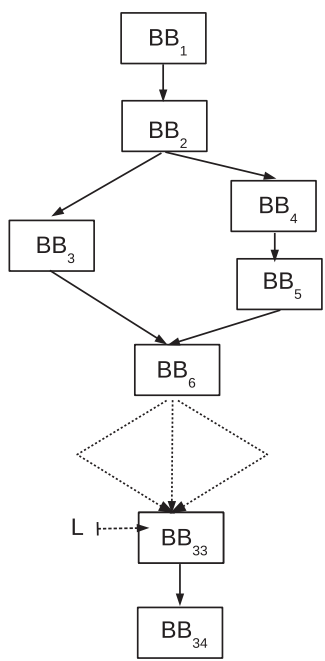

Fig. 5. Release of shared scratchpad.

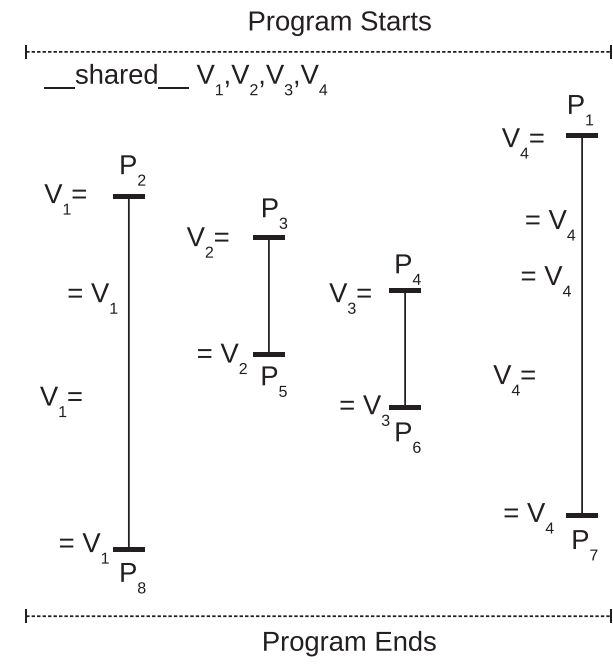

Fig. 6. Access ranges of scratchpad variables.

as one thread block, say $\mathrm{TB}_{0}$, starts accessing the shared scratchpad region, the other thread block, $\mathrm{TB}_{1}$, cannot access the shared scratchpad region and hence may have to wait until $\mathrm{TB}_{0}$ finishes execution.

Example 4.1. Consider the CFG in Figure 5, which is obtained for SRAD1 benchmark application (Table I). In the figure, the program point marked $\mathbf{L}$ corresponds to the last access to the shared scratchpad. Without compiler assistance, the shared scratchpad region can be released only at the end of the last basic block (Exit node of CFG) even though it is never accessed after L.

To promote the release of shared scratchpad region before the end of kernel execution, we introduce a new hardware instruction (PTX instruction) called relssp. Our proposed compiler optimization can place the relssp instructions in a kernel such that shared scratchpad memory is released as early as possible by each thread.

Example 4.2. Consider the scenario in Figure 6, where a kernel function declares four equal sized scratchpad variables $V_{1}$ to $V_{4}$. The figure also shows the regions of the kernel within which different variables are accessed. If $V_{1}$ and $V_{4}$ are allocated into shared scratchpad region, then the shared scratchpad region is accessed from program point $P_{1}$ to program point $P_{8}$. However, when $V_{2}$ and $V_{3}$ are allocated to shared scratchpad region, the shared region is accessed for a shorter duration, that is, from program point $P_{3}$ to program point $P_{6}$.

Note that the choice of allocation of scratchpad variables into shared and unshared scratchpad regions does not affect the correctness of the program but can affect the availability of the shared scratchpad region, and hence the effectiveness of sharing.

\section{COMPILER OPTIMIZATIONS}

In this section, we describe a compile time memory allocation scheme and an analysis to optimally place relssp instructions. The memory allocation scheme allocates scratchpad variables into shared and unshared region such that shared scratchpad variables are accessed only for a small duration during the run-time. In the presence of loops, where the number of iterations of loops are not computable at compile time, it is not possible 
to statically bound the number of instructions executed at run-time. Hence, we need to use approximate loop bounds. Any approximation is fine, since, as noted earlier, it only affects the effectiveness of sharing, but not the correctness. ${ }^{3}$

To simplify the description of the required analyses, we make the following assumptions:

-The control flow graph (CFG) for a function (kernel) has a unique Entry and a unique Exit node.

- There are no critical edges in the CFG. A critical edge is an edge whose source node

has more than one successor and the destination node has more than one predecessor.

These assumptions are not restrictive as any control flow graph can be converted to the desired form using a preprocessing step involving simple graph transformations: adding a source node, adding a sink node, and adding a node to split an edge [Khedker et al. 2009; Kam and Ullman 1976].

\subsection{Minimizing Shared Scratchpad Region}

Consider a GPU that uses scratchpad sharing approach such that two thread blocks involved in sharing can share a fraction $f<1$ of scratchpad memory. Assume that each SM in the GPU has $M$ bytes of scratchpad memory, the kernel that is to be launched into the SM has $N$ scratchpad variables, and each thread block of the kernel requires $M_{t b}$ bytes of scratchpad memory. We allocate a subset $S$ of scratchpad variables into shared scratchpad region such that: (1) The total size of the scratchpad variables in the set $S$ is equal to the size of shared scratchpad $\left(f \times M_{t b}\right)$, and (2) the region of access for variables in $S$ is minimal in terms of the number of instructions.

To compute the region of access for $S$, we define access range for a variable as follows:

Definition 5.1. Access Range of a Variable: A program point $\pi$ is in the access range of a variable $v$ if both the following conditions hold: (1) There is an access (definition or use) of $v$ on some path from Entry to $\pi$, and (2) there is an access of $v$ on some path from $\pi$ to Exit.

Intuitively, the access range of a variable covers every program point between the first access and the last access of the variable in an execution path. The access range for a variable can contain disjoint regions due to branches in the flow graph.

Definition 5.2. Access Range of a Set of Variables: A program point $\pi$ is in the access range of a set of variable $S$ if both the following conditions hold: (1) There is an access to a variable $v \in S$ on a path from Entry to $\pi$, and (2) there is an access to a variable $v^{\prime} \in S$ on a path from $\pi$ to Exit.

Example 5.1. Consider a kernel whose CFG is shown in Figure 7. The kernel uses three scratchpad variables $\mathrm{A}, \mathrm{B}$, and $\mathrm{C}$. Variable $\mathrm{A}$ is accessed in the region from basic block $B B_{1}$ to basic block $B B_{4}$. The start of basic block $B B_{2}$ is considered in access range of $\mathrm{A}$, because there is a path from Entry to start of $B B_{2}$ that contains an access of $\mathrm{A}$ (definition in $B B_{1}$ ), and there is a path from the start of $B B_{2}$ to Exit that contains the access of $\mathrm{A}$ (use in $\mathrm{BB}_{4}$ ).

Consider the set $S=\{\mathrm{B}, \mathrm{C}\}$. Basic block $B B_{4}$ is in access range of $S$, because there is a path from Entry to $B B_{4}$ containing the access of $\mathrm{B}$ (definition in $B B_{2}$ ), and there is a path from $B B_{4}$ to Exit containing the access of the $\mathrm{C}$ (use in $B B_{6}$ ).

\footnotetext{
${ }^{3}$ Profiling and user annotations can help in finding better approximations for the loop bounds. However, we have not used these in our current implementation.
} 


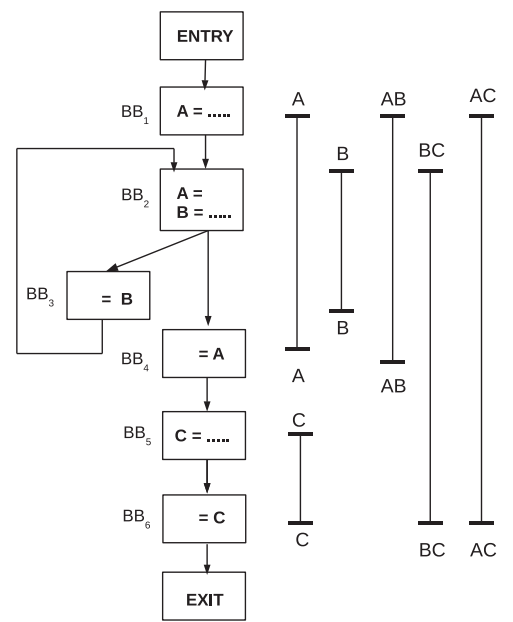

Table III. Access Ranges for Scratchpad Variables and Sets. $\mathbf{t}$ Denotes True, $\mathbf{f}$ Denotes False. Sets of Variables are Written as Concatenation of Variables. For Example, AB Denotes $\{A, B\}$

\begin{tabular}{r||c|c|c|c|c|c||c|c|c|c|c|c|c}
\hline \multicolumn{1}{c||}{} & \multicolumn{3}{c||}{ For Variables } & \multicolumn{4}{c|}{ For Sets of Variables } \\
\cline { 2 - 11 } Block & \multicolumn{2}{c|}{ IN } & A & C & A & B & C & AB & BC & CA & AB & BC & CA \\
\hline \hline Entry & f & f & f & f & f & f & f & f & f & f & f & f \\
BB1 & f & f & f & t & f & f & f & f & f & t & f & t \\
BB2 & t & t & f & t & t & f & t & t & t & t & t & t \\
BB3 & t & t & f & t & t & f & t & t & t & t & t & t \\
BB4 & t & f & f & f & f & f & t & t & t & f & t & t \\
BB5 & f & f & f & f & f & t & f & t & t & f & t & t \\
BB6 & f & f & t & f & f & f & f & t & t & f & f & f \\
Exit & f & f & f & f & f & f & f & f & f & f & f & f \\
\hline
\end{tabular}

Fig. 7. Access ranges of variables.

To compute the access ranges for a program, we need a forward analysis to find the first access of the scratchpad variables, and a backward analysis to find the last access of the scratchpad variables. We define these analyses formally using the following notations:

- IN $(B B)$ denotes the program point before the first statement of the basic block $B B$. OUT $(B B)$ denotes the program point after the last statement of $B B$.

-PRED $(B B)$ denotes the set of predecessors, and SUCC $(B B)$ denotes the set of successors of $B B$.

- PreIN $(v, B B)$ is true if there is an access to variable $v$ before $\operatorname{IN}(B B)$. PreOUT $(v, B B)$ is true if there is an access to the variable $v$ before OUT $(B B)$.

- PostIN $(v, B B)$ is true if there is an access to variable $v$ after $\operatorname{IN}(B B)$. PostOUT $(v, B B)$ is true if there is a access to variable $v$ after OUT $(B B)$.

- AccIN $(S, B B)$ is true if $\mathrm{IN}(B B)$ is in access range of a set of scratchpad variables $S$. $\operatorname{AccOUT}(S, B B)$ is true if OUT $(B B)$ is in access range of a set of scratchpad variables $S$.

The data flow equations to compute the information are ${ }^{4}$ :

$$
\begin{aligned}
& \text { PreOUT }(v, B B)=\left\{\begin{array}{l}
\text { true, if } B B \text { has an access of } v \\
\text { PrelN }(v, B B), \text { otherwise, }
\end{array}\right. \\
& \operatorname{PreIN}(v, B B)=\left\{\begin{array}{l}
\text { false, if } B B \text { is Entry block } \\
\bigvee_{B P \in \operatorname{PRED}(B B)} \operatorname{PreOUT}(v, B P), \text { otherwise, }
\end{array}\right. \\
& \text { PostIN }(v, B B)=\left\{\begin{array}{l}
\text { true, if } B B \text { has an access of } v \\
\text { PostOUT }(v, B B), \text { otherwise, }
\end{array}\right. \\
& \operatorname{PostOUT}(v, B B)=\left\{\begin{array}{l}
\text { false, if } B B \text { is Exit block } \\
\bigvee_{B S \in \operatorname{SUCC}(B B)} \operatorname{PostIN}(v, B S) \text {, otherwise. }
\end{array}\right.
\end{aligned}
$$

\footnotetext{
${ }^{4}$ The analysis can be extended easily to compute information at any point inside a basic block. We ignore it for brevity.
} 


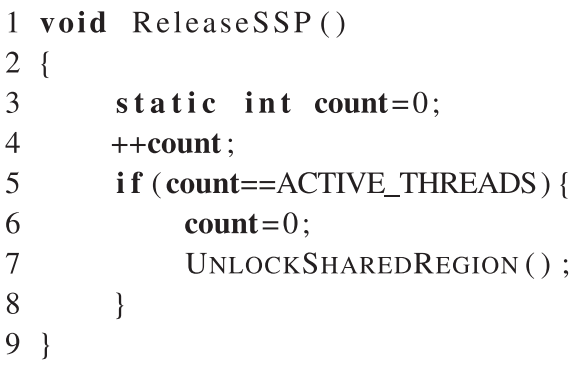

Fig. 8. Pseudocode of relssp instruction.

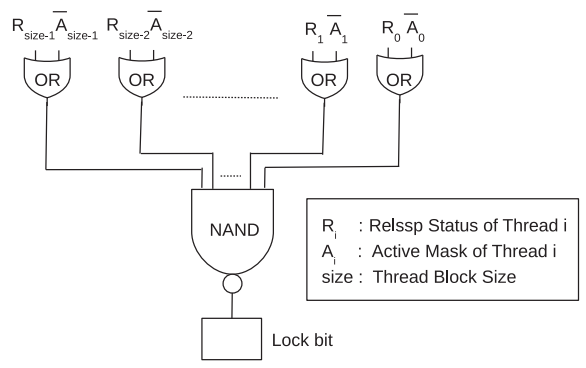

Fig. 9. Hardware implementation of relssp instruction.

We decide whether the access range of a set of scratchpad variables $S$ includes the points IN $(B B)$ and OUT $(B B)$ as:

$$
\begin{aligned}
\operatorname{AccIN}(S, B B) & =\left(\bigvee_{v \in S} \operatorname{PreIN}(v, B B)\right) \wedge\left(\bigvee_{v \in S} \operatorname{PostIN}(v, B B)\right), \\
\operatorname{AccOUT}(S, B B) & =\left(\bigvee_{v \in S} \operatorname{PreOUT}(v, B B)\right) \wedge\left(\bigvee_{v \in S} \operatorname{PostOUT}(v, B B)\right) .
\end{aligned}
$$

Example 5.2. Table III shows the program points in the access ranges of scratchpad variables for CFG of Figure 7. The table also shows the program points in the access ranges of sets of two scratchpad variables each.

Let $S V$ denote the set of all scratchpad variables. For every subset $S$ of $S V$ having a total size equal to the size of shared scratchpad memory, our analysis counts the total number of instructions in the access range of S. Finally, the subset that has the minimum count is selected for allocation in the shared scratchpad memory.

Example 5.3. Consider once again the CFG in Figure 7. For simplicity, assume that all the variables have equal sizes, and each basic block contains the same number of instructions. Consider a scratchpad sharing approach that can allocate only two of the variables into the shared scratchpad region. From the CFG, and from Table III, it is clear that when A and B are allocated into shared scratchpad memory, the shared region is smaller, compared to when either $\{B, C\}$ or $\{A, C\}$ are allocated in the shared region.

\subsection{Implementation of relssp Instruction}

In scratchpad sharing approach, a shared thread block acquires a lock before accessing shared scratchpad region and unlocks it only after finishing its execution. This causes a delay in releasing the shared scratchpad, because the thread block holds the scratchpad memory till the end of its execution, even though it has finished accessing shared region.

To minimize the delay in releasing the shared scratchpad, we propose a new instruction, called relssp, in PTX assembly language. The semantics of relssp instruction is to unlock the shared region only when all active threads ${ }^{5}$ within a thread block finished executing the shared region. Figure 8 shows the pseudo code for relssp instruction. The RELEASESSP() procedure maintains count, an integer initialized to zero. When an active thread within a thread block executes a relssp instruction, it increments

\footnotetext{
${ }^{5}$ In GPGPU-Sim, additional threads are padded to a thread block to make the number of threads in the thread block a multiple of the warp size. We call these additional threads used for padding as inactive, and the remaining threads as active.
} 
the count value. When all active threads of a thread block execute relssp instruction (Line 5, when count equals ACTIVE_THREADS), the shared region is unlocked by invoking UnLOCKSHAREDREGION(). The unlock procedure releases the shared scratchpad region by resetting the lock variable. The execution of relssp by a thread block that does not access shared scratchpad region has no effect.

It is clear that count in Figure 8 has to be a shared variable, hence a software implementation will require to manage critical section. The same algorithm, however, can be efficiently implemented in hardware circuit as shown in Figure 9. The $i$ th thread within a thread block is associated with an active mask $\left(A_{i}\right)$ and a release bit $\left(R_{i}\right)$. The mask $A_{i}$ is set if the $i$ th thread is active. When this thread executes relssp instruction, the release bit $\left(R_{i}\right)$ gets set. The shared scratchpad region is unlocked only when all the active threads in a thread block execute relssp instruction (the lock bit, i.e., the output of NAND gate becomes 0 in Figure 9). In other words, shared scratchpad region is unlocked if $\forall i A_{i} \rightarrow R_{i}$ is true.

\subsection{Algorithm for Optimal Placement of relssp Instruction}

In Section 5.2, we introduced a new instruction to release the shared scratchpad memory. In this section, we discuss a compile-time analysis for optimal insertion of relssp instruction in the program. We insert a relssp instruction at a program point $\pi$ such that the following conditions are met:

(1) Safety: The relssp instruction must be executed by each active thread within a thread block, and it must be executed after last access to shared scratchpad memory.

(2) Optimality: The relssp instruction must be executed by each active thread exactly once.

Condition (1) (Safety) ensures that shared scratchpad is eventually released by a thread block, since the instruction is executed by all the threads of a thread block. Also, it guarantees that shared scratchpad is released only after a thread block has completed using it. Whereas, Condition (2) (Optimality) avoids redundant execution of relssp instruction.

In the scratchpad sharing, a thread block releases the shared scratchpad memory after completing its execution, hence it is equivalent to having a relssp instruction placed at the end of the program, which guarantees both the conditions, albeit at the cost of delay in releasing the shared scratchpad. A simple improvement that promotes early release of shared scratchpad memory and ensures both the conditions is to place the relssp instruction at a basic block $B B_{\text {postdom }}$ where $B B_{\text {postdom }}$ is a common post dominator of those basic blocks having the last accesses to the shared scratchpad memory along different paths. Further, $B B_{\text {postdom }}$ should dominate Exit, that is, it should be executed in all possible execution paths. As the following example shows, this strategy, though an improvement over placing relssp in Exit, may also result in delaying the release of shared scratchpad memory.

Example 5.4. Consider a CFG shown in Figure 10. Assume that $L_{1}, L_{2}$ denote the program points that correspond to the last accesses to shared scratchpad memory. Since relssp instruction is to be executed by all the threads of thread block, it can be placed at the post dominator of the basic blocks $B B_{3}$ and $B B_{9}$, that is, program point marked $\pi$ in $B B_{12}$, which is visible to all threads. However, this delays the release of shared scratchpad. Consider a thread that takes a path along $B B_{9}$, it can execute relssp immediately after executing the last access to shared region (shown as $O P T_{3}$ in the figure). It executes relssp at program point $\pi$. Similarly, when a thread takes a path along the basic block $B B_{4}$, it releases the shared scratchpad at $\pi$ even though 


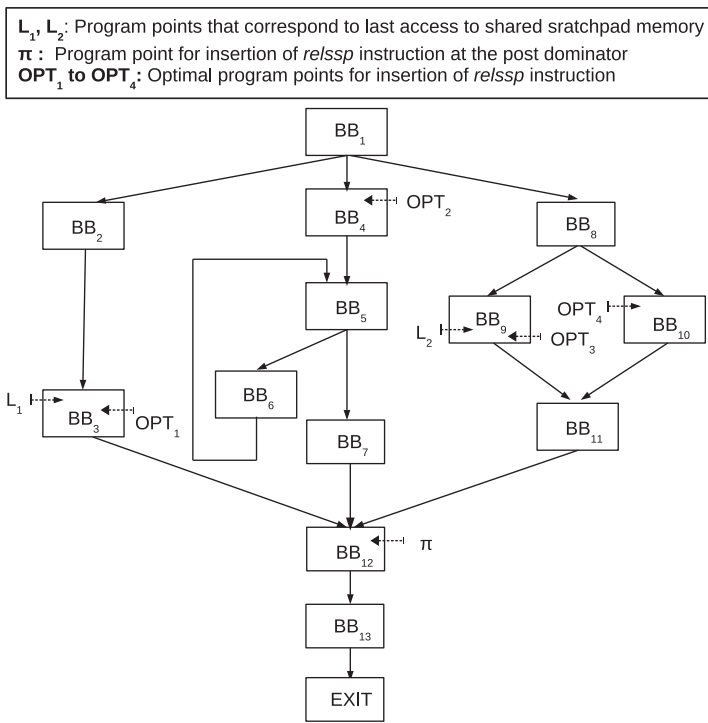

Fig. 10. Possible insertion points for relssp.

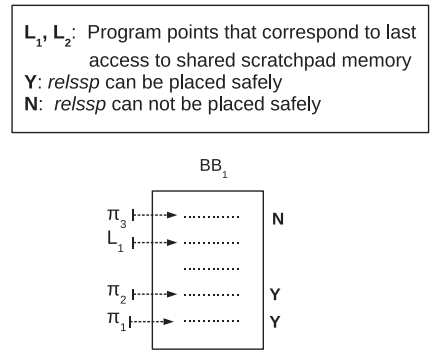

(a)

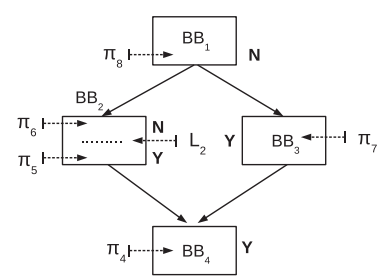

(b)

Fig. 11. Scenarios for optimal insertion of relssp.

it does not access any shared scratchpad in that path. The scratchpad can be released at program point $O P T_{2}$ in $B B_{4}$.

As is clear from the above example, placement of relssp instruction has an effect on the availability of shared scratchpad memory. Intuitively, a safely placed relssp instruction at a program point $\pi$ can be moved to a previous program point $\pi^{\prime}$ in the same basic block provided the intervening instructions do not access shared scratchpad. The movement of relssp from a basic block $B B$ to predecessor $B B^{\prime}$ is possible provided every other successor of $B B^{\prime}$ also does so.

Example 5.5. Figure 11(a) shows a basic block $B B_{1}$, which has the last access to the shared scratchpad memory at $L_{1}$. In this block, if the relssp instruction can be placed safely at the program point $\pi_{1}$, then it can be moved to $\pi_{2}$, since there is no access to shared scratchpad memory between $\pi_{1}$ and $\pi_{2}$. However, it cannot be moved to the program point $\pi_{3}$ within the same basic block, because it violates safety (Condition 1 ).

Consider another scenario shown in Figure 11(b), basic block $B B_{2}$ has the last access to shared memory at $L_{2}$, and basic blocks $B B_{1}, B B_{3}$, and $B B_{4}$ do not access any scratchpad memory. If the relssp instruction can be placed safely at $\pi_{4}$ in $B B_{4}$, then it can be moved to a program point $\pi_{5}$ and $\pi_{7}$ in the basic blocks $B B_{2}$ and $B B_{3}$, respectively. However, it cannot be moved to program point $\pi_{6}$ in $B B_{2}$ and $\pi_{8}$ in $B B_{1}$, since it violates of Condition 1. Also, the relssp instruction can not be moved from $\pi_{7}$ in $B B_{3}$ to $\pi_{8}$ in $B B_{1}$, since the basic block $B B_{2}$, which is a successor of $B B_{1}$, does not allow the relssp instruction to be placed at $\pi_{8}$.

We now formalize these intuitions into a backward data flow analysis. The notations used are:

- IN $(B B)$ denotes the program point before the first statement of the basic block $B B$.

OUT $(B B)$ denotes the program point after the last statement of $B B$. 
- SafelN $(B B)$ is true if the relssp instruction can be safely placed at $\operatorname{IN}(B B)$, and SafeOUT $(B B)$ is true if the relssp instruction can be safely placed at OUT $(B B)$. $-\mathrm{INS}_{\pi}$, if true, denotes that relssp will be placed at program point $\pi$ by the analysis.

The data flow equations are:

$$
\begin{gathered}
\text { SafeIN }(B B)=\left\{\begin{array}{c}
\text { false, if } B B \text { has shared } \\
\text { scratchpad access } \\
\text { SafeOUT }(B B), \text { otherwise, }
\end{array}\right. \\
\text { SafeOUT }(B B)=\left\{\begin{array}{c}
\text { true, if BB is Exit block } \\
\bigwedge_{B S \in \operatorname{SUCC}(B B)} \text { SafelN }(B S), \text { otherwise. }
\end{array}\right.
\end{gathered}
$$

The above equations compute the program points where relssp can be placed safely. For a basic block $B B$, OUT $(B B)$ is an optimal place for relssp instruction, if relssp can be placed safely at OUT $(B B)$, and it can not be moved safely to its previous program point in the basic block, that is, $\mathrm{IN}(B B)$ is false. This is computed as

$$
\operatorname{INS}_{\mathrm{OUT}(B B)}=\text { SafeOUT }(B B) \wedge \neg(\text { SafeIN }(B B)) \text {. }
$$

Similarly, IN $(B B)$ is an optimal point for relssp instruction, when the instruction can not be moved to its predecessors. ${ }^{6}$ This can be computed as

$$
\mathrm{INS}_{\mathrm{IN}(B B)}=\neg\left(\bigwedge_{B P \in \operatorname{PRED}(B B)} \operatorname{SafeOUT}(B P)\right) \wedge \text { SafeIN }(B B) .
$$

Equations (1) and (2) together, along with the absence of critical edges, ensure the optimality condition that each thread executes the relssp instruction exactly once. Thus, our compiler optimizations help in progressing the shared thread blocks that are present in each SM by releasing shared scratchpad and minimizing the access to shared scratchpad region. Hence, the shared thread blocks in each SM finish their execution early. Subsequently, new shared thread blocks are launched early. Thus, a kernel can finish its execution early.

\section{REQUIREMENTS FOR SCRATCHPAD SHARING}

\subsection{Hardware Requirements}

Figure 12 shows the modified GPU architecture to implement scratchpad sharing. Our approach requires two modifications to scheduler unit in the SM. The first change is to the warp scheduler, which uses OWF optimization. The second change is the inclusion of resource access unit, which follows the scratchpad access mechanism as discussed in Section 3. The resource access unit requires the following additional storage:

(1) Each SM requires a bit (shown as ShSM in Figure 12) to indicate whether the scratchpad sharing is enabled for it. This bit is set when the number of thread blocks launched using our approach is more than that of baseline approach.

(2) Every thread block involved in sharing stores the id of its partner thread block in the $S h T B$ table. If a thread block is in unsharing mode, then -1 is stored. For $T$ thread blocks in the SM, we need a total of $T \log _{2}(T+1)$ bits.

(3) Each warp requires a bit to specify if it is an owner warp.

\footnotetext{
${ }^{6}$ Absence of critical edges guarantees that the instruction can either be moved to all predecessors or to none.
} 


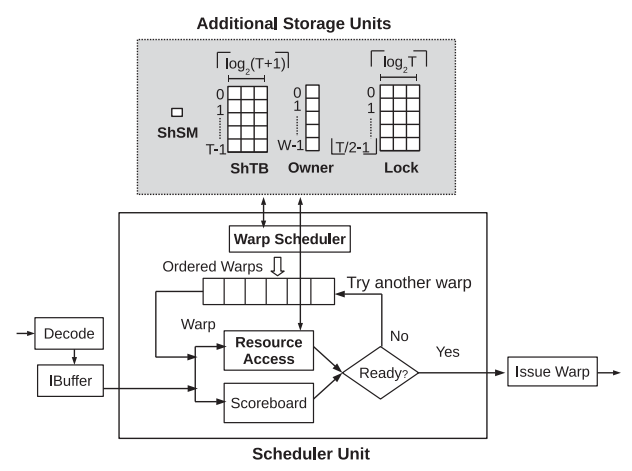

Fig. 12. Modified architecture for scratchpad sharing.

(4) For each pair of shared thread blocks, a lock variable is needed to access shared scratchpad memory. This variable is set to the id the thread block that acquired shared scratchpad memory. For $T$ thread blocks, there are at most $\lfloor T / 2\rfloor$ pairs of sharing thread blocks in the SM. This requires $\left\lfloor\frac{T}{2}\right\rfloor\left\lceil\left(\log _{2} T\right)\right\rceil$ bits in the SM.

If a GPU has $N$ SMs and allows a maximum of $T$ thread blocks and $W$ warps per SM, then the number of additional bits required is $\left(1+T \log _{2}(T+1)+W+\left\lfloor\frac{T}{2}\right\rfloor\left\lceil\left(\log _{2} T\right)\right\rceil\right) * N$. For the architecture, we used for simulation (shown in Table II), the overhead is 209 bits per SM. In addition, each scheduler unit in the SM requires two comparator circuits and one arithmetic circuit to set the lock (See Figure 3).

\subsection{Analysis of Compiler Optimizations}

The dataflow analyses to compute definitions and usages of scratchpad variables (Section 5.1) are bit-vector data flow analyses [Khedker et al. 2009]. For a kernel with $n$ scratchpad variables and $m$ nodes (basic blocks) in the flow graph, the worst case complexity is $O\left(n \times m^{2}\right)$ (assuming set operations on $n$ bit-wide vectors take $O(n)$ time).

The computation of access ranges for sets of variables may require analyzing all $O\left(2^{n}\right)$ subsets in the worst case, where the largest size of a subset is $O(n)$. Thus, given the usage and definitions at each program point in the kernel, computation of AccIN and AccIN requires $O\left(m \times n \times 2^{n}\right)$ time. Therefore, the total time complexity is $O\left(n \times m^{2}+m \times n \times 2^{n}\right)$. Since the number of scratchpad variables in a kernel function is small (typically, $n \leq 10$ ), the overhead of the analysis is practical.

Our approach inserts relssp instructions in a CFG such that relssp is called exactly once along any execution path. In the worst case, all nodes in a CFG (except Entry and Exit blocks) might fall along different paths from Entry to Exit. Hence, the worst case number of relssp inserted is $O(m)$.

\section{EXPERIMENTAL EVALUATION}

We implemented the proposed scratchpad sharing approach and integrated relssp instruction in GPGPU-Sim V3.x [GPGPUSIM 2014] simulator. We implemented the compiler optimizations in PTX assembly [PTX 2014] using Ocelot [Diamos et al. 2010] framework. The baseline architecture that we used for comparing our approach is shown in Table II. We evaluated our approach on several kernels from CUDASDK [CUDA-SDK 2014], GPGPU-Sim [Bakhoda et al. 2009], and Rodinia [Che et al. 2009] benchmark suites.

Depending on the amount and the last usage of the shared scratchpad memory by the applications, we divided the benchmark applications into three sets. Set- 1 and 
Table IV. Set-3 Benchmarks: The Number of Thread Blocks is Not Limited by Scratchpad Memory

\begin{tabular}{llll}
\hline \hline Benchmark & \multicolumn{1}{c}{ Application } & \multicolumn{1}{c}{ Kernel } & \multicolumn{1}{c}{ Limited by } \\
\hline GPGPU-SIM & BFS & Kernel & Threads, Registers \\
RODINIA & b+tree & findRangeK & Registers \\
CUDA-SDK & dct8x8_5 (DCT5) & CUDAkernel1DCT & Blocks \\
RODINIA & gaussian & FAN1 & Threads \\
GPGPU-SIM & NN & executeSecondLayer & Blocks \\
\hline
\end{tabular}

Set-2 (Table I) consists of applications whose number of resident thread blocks are limited by scratchpad memory. For Set-1, the applications do not access scratchpad memory till towards the end of their execution, while for Set-2, the applications access scratchpad memory till towards the end of their execution. The introduction of relssp instruction is expected to give benefit over our earlier approach [Jatala et al. 2016a] only for Set-1 applications. Set-3 benchmarks (Table IV) consist of applications whose number of thread blocks are not limited by scratchpad memory, but by some other parameter. These are included to show that our approach does not negatively affect the performance of applications that are not limited by scratchpad memory.

For each application in Set-1 and Set-2 benchmarks, Table I shows the kernel that is used for evaluation, the number of the scratchpad variables declared in each kernel, the amount of the scratchpad memory required for each thread block, and the thread block size. Some applications in Set-1 and Set-2 benchmarks are modified to make sure that the number of thread blocks is limited by scratchpad memory, thus making scratchpad sharing approach applicable. These changes increase the scratchpad memory requirement per thread block and are described in details in Jatala et al. [2016b]. For Set-3 benchmarks, Table IV shows the cause of limitation on the number of thread blocks. The causes include the limit on the number of registers, the maximum limit on the number of resident thread blocks, and the maximum limit on the number of resident threads.

We compiled all the applications using CUDA 4.0 and simulated them using the GPGPU-Sim simulator. CUDA 4.0 is used, since GPGPU-Sim and Ocelot do not support CUDA 5.0 and above. We use a threshold $(t)$ to configure the amount of scratchpad sharing. If each thread block requires $R_{t b}$ amount of scratchpad memory, then we allocate $R_{t b}(1+t)$ for each pair of shared thread blocks, in which we allocate $R_{t b}(1-t)$ as shared scratchpad memory. We analyzed the benchmark applications for various threshold values and choose the value $t$ as 0.1 (i.e., $90 \%$ scratchpad is shared among pair of thread blocks) to give the maximum benefit. The details of the experiments to choose $t$ are given in our technical report [Jatala et al. 2015]. For any loop with non-constant bounds, we assumed a bound of 1024 on the number of iterations of the loop.

We measure the performance of our approach using the following metrics:

(1) The number of the resident thread blocks launched in the SMs. This is a measure of the amount of thread level parallelism present in the SMs.

(2) The number of instructions executed per shader core clock cycle (IPC). This is a measure of the throughput of the GPU architecture.

(3) The number of simulation cycles that an application takes to complete its execution. This is a measure of the performance of the benchmark applications.

\subsection{Analysis of Set-1 and Set-2 Benchmarks}

We use Unshared-LRR to denote the baseline unsharing approach, Shared-OWF to denote our scratchpad sharing approach with OWF scheduler, and Shared-OWF-OPT 


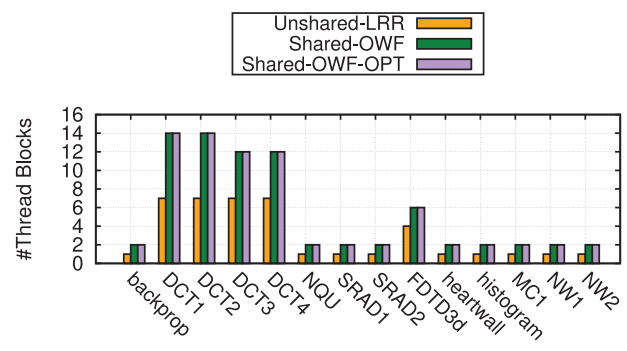

Fig. 13. Comparing the resident thread blocks.

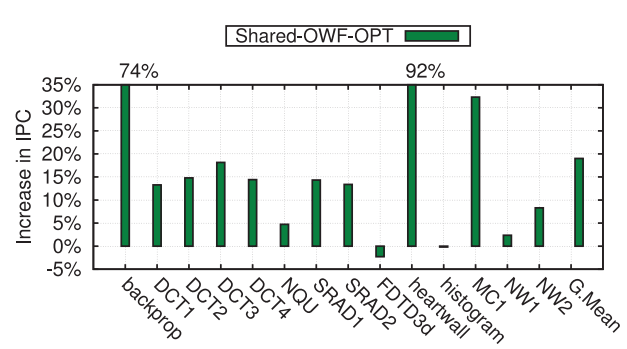

Fig. 14. Comparing the IPC.

to denote the scratchpad sharing approach that includes OWF scheduler and compiler optimizations.

7.1.1. Comparing the Number of Resident Thread Blocks. Figure 13 shows the number of thread blocks for the three approaches. For applications DCT1 and DCT2, Unshared$L R R$ launches 7 thread blocks in the SM according to the amount of scratchpad memory required by their thread blocks. Shared-OWF launches 14 thread blocks in the SM, where each of the 7 additional thread blocks share scratchpad memory with other resident thread blocks. For DCT3 and DCT4 applications, Unshared-LRR launches 7 thread blocks in the SM, whereas Shared-OWF launches 12 thread blocks in the SM, such that the additional 5 thread blocks share scratchpad memory with the existing 5 thread blocks; while the remaining 2 existing thread blocks in the SM do not share scratchpad memory with any other thread block. For FDTD3d, Shared-OWF launches 2 additional thread blocks in the SM when compared to Unshared-LRR, which share scratchpad memory with other 2 resident thread blocks. For the remaining applications, Unshared-LRR launches 1 thread block, whereas Shared-OWF launches 1 additional thread block in the SM, which shares scratchpad memory with the existing thread block. Note that the number of thread blocks launched by Shared-OWF-OPT is exactly same as that of Shared-OWF. This is expected, since the number of additional thread blocks launched by scratchpad sharing approach depends on two parameters: (1) the amount of scratchpad sharing, and (2) the amount of scratchpad memory required by a thread block; and our compiler optimizations do not affect either of these parameters.

7.1.2. Performance Comparison. Figure 14 compares the performance of Shared-OWF$O P T^{7}$ in terms of the number of instructions executed per cycle (IPC) with that of Unshared-LRR. We observe a maximum improvement of $92.17 \%$ and an average (Geometric Mean, shown as G. Mean in the figure) improvement of $19 \%$ with Shared-OWF$O P T$. The maximum benefit of $92.17 \%$ is for heartwall, because the additional thread blocks launched by Shared-OWF-OPT do not access the shared scratchpad region. Hence all the additional thread blocks make progress without waiting for corresponding shared thread blocks. $M C 1$ improves by $32.32 \%$, because additional thread blocks launched in the SM make significant progress before accessing shared scratchpad region. backprop shows an improvement of $74.2 \%$, it leverages both scratchpad sharing and the compiler optimizations to perform better. The improvements in SRAD1 and $S R A D 2$ applications are largely due to the compiler optimizations. FDTD3d slows down $(-2.29 \%)$ with Shared-OWF-OPT due to more number of L1 and L2 cache misses when compared to Unshared-LRR. histogram does not benefit from sharing, since the thread

\footnotetext{
${ }^{7}$ IPC for Shared-OWF-OPT also takes into account the extra instructions inserted by the compiler optimizations. The absolute values for IPC are shown in Jatala et al. [2016b].
} 
Table V. Comparing the Number of Simulated Instructions

\begin{tabular}{lrrrrr}
\hline \hline Benchmark & Threads & Unshared-LRR (U) & Shared-OWF (S) & Shared-OWF-OPT (SO) & Difference (SO - U) \\
\hline backprop & $1,048,576$ & $131,203,072$ & $131,203,072$ & $133,234,688$ & $2,031,616$ \\
DCT1 & 32,768 & $9,371,648$ & $9,371,648$ & $9,404,416$ & 32,768 \\
DCT2 & 32,768 & $9,502,720$ & $9,502,720$ & $9,535,488$ & 32,768 \\
DCT3 & 32,768 & $11,255,808$ & $11,255,808$ & $11,304,960$ & 49,152 \\
DCT4 & 32,768 & $11,157,504$ & $11,157,504$ & $11,206,656$ & 49,152 \\
NQU & 24,576 & $1,282,747$ & $1,282,747$ & $1,331,515$ & 48,768 \\
SRAD1 & $4,161,600$ & $756,433,955$ & $756,433,955$ & $760,595,555$ & $4,161,600$ \\
SRAD2 & $4,161,600$ & $450,077,975$ & $450,077,975$ & $454,239,575$ & $4,161,600$ \\
FDTD3d & 144,384 & $5,549,531,392$ & $5,549,531,392$ & $5,549,820,160$ & 288,768 \\
heartwall & 17,920 & $11,280,920$ & $11,280,920$ & $11,316,760$ & 35,840 \\
histogram & 46,080 & $893,769,168$ & $893,769,168$ & $893,861,328$ & 92,160 \\
MC1 & 3,008 & $2,881,568$ & $2,881,568$ & $2,887,584$ & 6,016 \\
NW1 & 3,184 & $5,580,458$ & $5,580,458$ & $5,583,642$ & 3,184 \\
NW2 & 3,168 & $5,561,919$ & $5,561,919$ & $5,565,087$ & 3,168 \\
\hline
\end{tabular}

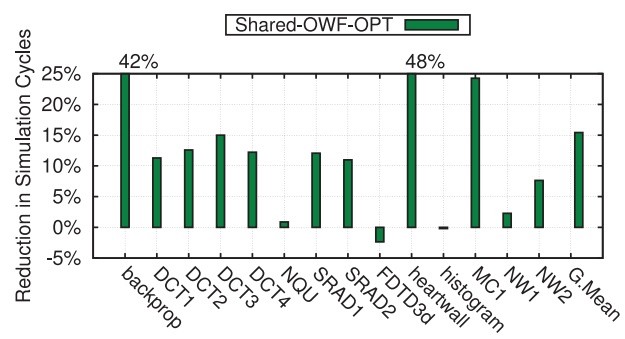

Fig. 15. Comparing the simulation cycles.

blocks start accessing shared scratchpad region early in the execution, causing one of the blocks from each sharing pair to wait for the lock.

7.1.3. Overhead of Relssp Instruction. Table V shows the run-time overhead of inserting relssp instruction. We report sum of the number of instructions executed by all threads for Unshared-LRR, Shared-OWF, and Shared-OWF-OPT. We also report the number of threads launched.

From the table, we observe that the number of instructions executed by Unshared$L R R$ and Shared-OWF is same. This is because Shared-OWF does not insert relssp instruction, and hence the input PTX assembly is not altered. Shared-OWF-OPT increases number of executed instructions as it inserts relssp and, in some cases, GOTO instruction to split critical edges. For the applications DCT1, DCT2, SRAD1, SRAD2, $N W 1$, and NW2, the number of additionally executed instructions (shown as Difference (SO-U) in the table) is equal to number of threads, because Shared-OWF-OPT inserts only the relssp instruction. Further, each thread executes relssp exactly once. For FDTD3d, heartwall, histogram, and MC1 applications, the number of additional instructions executed by Shared-OWF-OPT is twice that of number of threads. For these applications, each thread executes two additional instructions, that is, one relssp instruction and one GOTO instruction for splitting a critical edge. For backprop, DCT3, $D C T 4$, and NQU applications, some threads take a path that has two additional instructions (GOTO and relssp), while other threads take the path that has one additional relssp instruction.

7.1.4. Reduction in Simulation Cycles. In Figure 15, we observe a maximum reduction of $47.8 \%$ and an average reduction of $15.42 \%$ in the number of simulation cycles for Shared-OWF-OPT when compared to Unshared-LRR. Recall that Shared-OWF-OPT causes applications to execute more number of instructions (Table V). These extra 


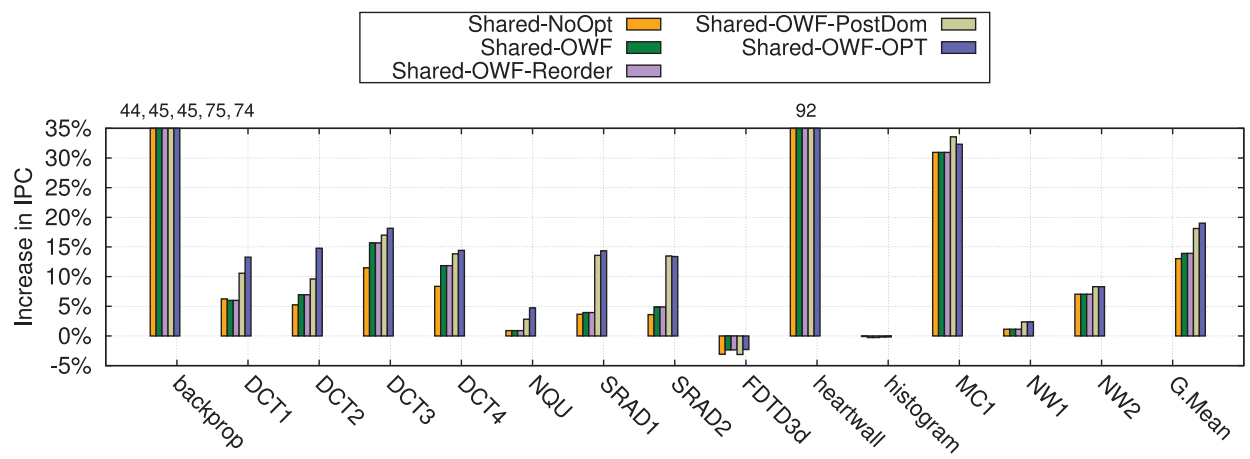

Fig. 16. Performance analysis of optimizations.

instructions are also counted while computing the simulation cycles for Shared-OWF$O P T$.

7.1.5. Effectiveness of Optimizations. Figure 16 shows the effectiveness of our optimizations with scratchpad sharing. We observe that all applications, except FDTD-3d and histogram, show some benefit with scratchpad sharing even without any optimizations (shown as Shared-NoOpt). With OWF scheduling (Shared-OWF), applications improve further, because OWF schedules the resident warps in a way that the nonowner warps help in hiding long execution latencies. For our benchmarks, minimizing shared scratchpad region (shown as Shared-OWF-Reorder) does not have any noticeable impact. This is because (a) Most applications declare only a single scratchpad variable (Table I) in their kernel, hence the optimization is not applicable (there is only one possible order of scratchpad variable declarations); and (b) for the remaining applications, the scratchpad declarations are already ordered in the optimal fashion, that is, the access to shared scratchpad region is already minimal.

The addition of relssp instruction at the postdominator and at the optimal places is denoted as Shared-OWF-PostDom and Shared-OWF-OPT, respectively. All Set-1 applications improve with either of these optimizations, because the relssp instruction helps in releasing the shared scratchpad memory earlier. For backprop and SRAD2 applications, Shared-OWF-PostDom is better than Shared-OWF-OPT, because the threads in backprop execute one additional GOTO instruction with Shared-OWF-OPT (Shared$O W F$-PostDom does not require critical edge splitting). SRAD2 has more number of stall cycles with Shared-OWF-OPT as compared to Shared-OWF-PostDom. For most of the other benchmarks, Shared-OWF-OPT performs better as it can push relssp instruction earlier than with Shared-OWF-PostDom, thus releasing shared scratchpad earlier allowing for more thread level parallelism.

As expected, Set-2 applications do not show much benefit with Shared-OWF-PostDom or Shared-OWF-OPT, since they access shared scratchpad memory till toward the end of their execution. Hence, both the optimizations insert relssp instruction in the Exit block in the CFGs. The application heartwall does not use shared scratchpad memory and hence it shows maximum benefit even without the insertion of relssp instruction.

7.1.6. Progress of Shared Thread Blocks. Figure 17 shows the effect of compiler optimizations by analyzing the progress of shared thread blocks through shared and unshared scratchpad regions. In the figure, $N o O p t$ denotes the default scratchpad sharing when no optimizations are applied on an input kernel. Minimize denotes the scratchpad sharing, which executes an input kernel having minimum access to shared scratchpad region. PostDom and OPT use our modified scratchpad sharing approach that 


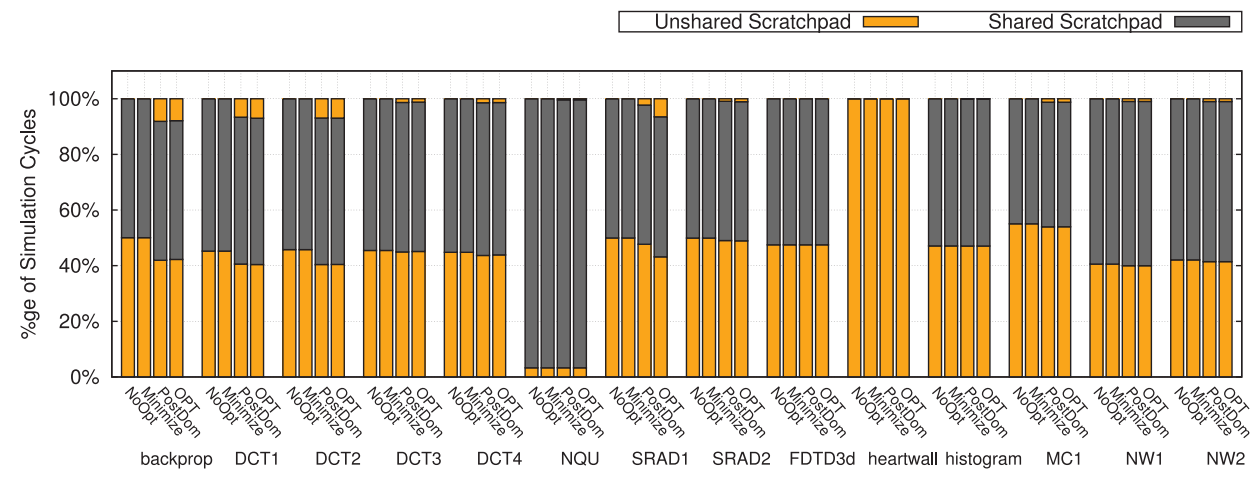

Fig. 17. Progress of shared thread blocks.

execute an input kernel with additional relssp instructions placed at post dominator and optimal places (Section 5.3), respectively. In the figure, we show the percentage of simulation cycles spent in unshared scratchpad region (before acquiring shared scratchpad), shared scratchpad region, and unshared scratchpad region again (after releasing the shared scratchpad), respectively. We observe that shared thread blocks in all the applications access unshared scratchpad region before they start accessing shared scratchpad memory. Hence, all the shared thread blocks can make some progress without wait. This progress is the main reason for the improvements seen with scratchpad sharing approach.

An interesting case to consider in Figure 17 is the application heartwall, where none of the shared thread blocks accesses shared scratchpad memory. However, note that this is run-time information, specific to the particular inputs used for the benchmark. In this case, there is no scope for compiler optimizations to further improve the progress of shared thread blocks. It is important to note that even though shared scratchpad memory is not accessed for the particular execution, it is used in the program code under an if-then-else condition. Thus, it is not possible to eliminate the shared scratchpad at compile-time.

It is clear from the figure that Minimize does not affect DCT1, DCT2, DCT3, DCT4, FDTD3d, histogram applications, because the kernels in these applications declare single scratchpad variable. For the remaining applications, Minimize has same effect as that of NoOpt, because the default input PTX kernel already accesses the shared scratchpad variables such that access to shared scratchpad is minimum. We also observe that PostDom and OPT approaches improve only those applications that spend considerable simulation cycles in unshared scratchpad region after last access to shared scratchpad region. We also observe that in NoOpt and Minimize approaches owner thread blocks keep shared scratchpad locked till the end of their execution. Hence, non-owner thread blocks wait for lock in the unshared scratchpad region for longer periods. However, with PostDom and AllOpt approaches, owner thread blocks release the shared scratchpad memory early using the relssp instruction, hence the non-owner thread blocks can start accessing the shared scratchpad early, minimizing the waiting time. Thus, we see an overall performance improvement.

7.1.7. Comparison with Different Schedulers. Figure 18 shows the effect of using different scheduling policies. The performance of Shared-OWF-OPT approach is compared with the baseline unshared implementation that uses greedy then old (GTO) and two-level scheduling policies, respectively. We observe that Shared-OWF-OPT approach shows an average improvement of $17.73 \%$ and $18.08 \%$ with respect to unshared GTO and 


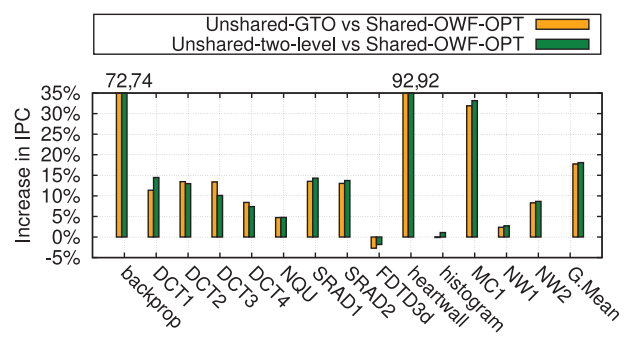

Fig. 18. Improvement in IPC for shared-OWF$O P T$ w.r.t. baseline having (a) GTO, (b) two-level scheduler.

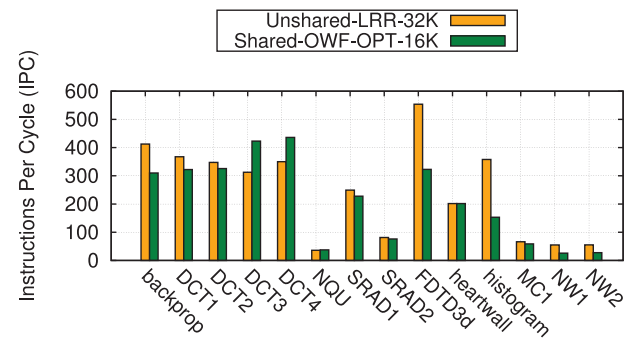

Fig. 19. Comparison with unshared-LRR that uses twice the scratchpad memory.

two-level scheduling policies, respectively. The application FDTD3d degrade with our approach when compared to the baseline with either GTO scheduling or two-level scheduling, since it has more number of L1 and L2 cache misses with sharing. The application histogram degrades with sharing when compared to the baseline with GTO scheduling because of a greater number of L1 misses. However, histogram with sharing performs better than the baseline with two-level policy.

7.1.8. Resource Savings. Figure 19 compares the IPC of Shared-OWF-OPT with Unshared-LRR that uses twice the amount of scratchpad memory on GPU. We observe that DCT3, DCT4, NQU, and heartwall show improvement with Shared-OWF-OPT over Unshared-LRR even with half the scratchpad memory. This is because sharing helps in increasing the TLP by launching additional thread blocks in each SM. The applications $D C T 1, D C T 2, S R A D 1, S R A D 2$, and $M C 1$ applications perform comparable with both the approaches. For the remaining applications, Unshared-LRR with double scratchpad memory performs better than sharing, since a greater number of thread blocks are able to make progress with the former.

\subsection{Analysis of Set-3 Benchmarks}

Performance analysis of Set-3 benchmarks is shown in the Figure 20. Recall that the number of thread blocks launched by these applications is not limited by the scratchpad memory. We observe that the performance of the applications with Unshared$L R R$, Shared-LRR, and Shared-LRR-OPT is exactly the same. For Set-3 applications all thread blocks are launched in unsharing mode. Hence Shared-LRR behaves exactly same as Unshared-LRR. Since these applications do not use any shared scratchpad memory, our compiler optimizations do not insert relssp instruction in their PTX code. Hence, the number of instructions executed by the Shared-LRR-OPT approach is same as that of Shared-LRR. Similarly, we see that Unshared-GTO, Shared-GTO, and Shared-GTO-OPT behave exactly the same. However, with OWF optimization, Shared$O W F$ and Shared-OWF-OPT is comparable to the Unshared-GTO, because OWF optimization arranges the resident warps according to the owner. Since all the the thread blocks own their scratchpad memory, they are sorted according to the dynamic warp id. Hence, they perform comparable to Unshared-GTO. The performances with Shared$O W F$ and Shared-OWF-OPT are the same, because the compiler optimizations do not insert any relssp instruction.

\subsection{Additional Experiments}

7.3.1. Performance Comparison with Other Configurations. To further verify the effectiveness of our approach, we evaluated it on two GPU configurations that use scratchpad memory of size 48 and 64KB per SM (Table VII). The scratchpad memory and thread 


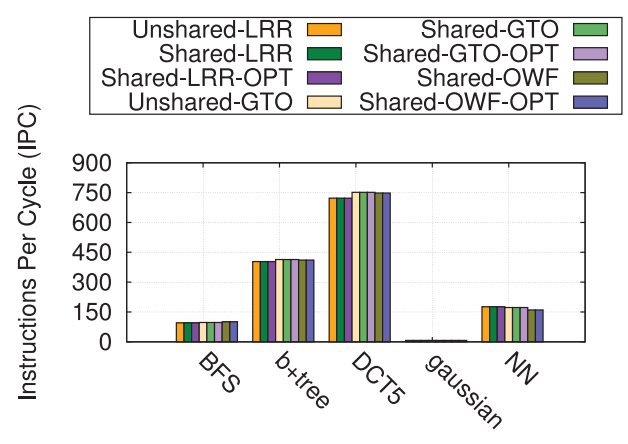

Fig. 20. IPC comparison of Set-3 benchmarks.

Table VI. Additional Benchmarks That Are Limited by Scratchpad Memory

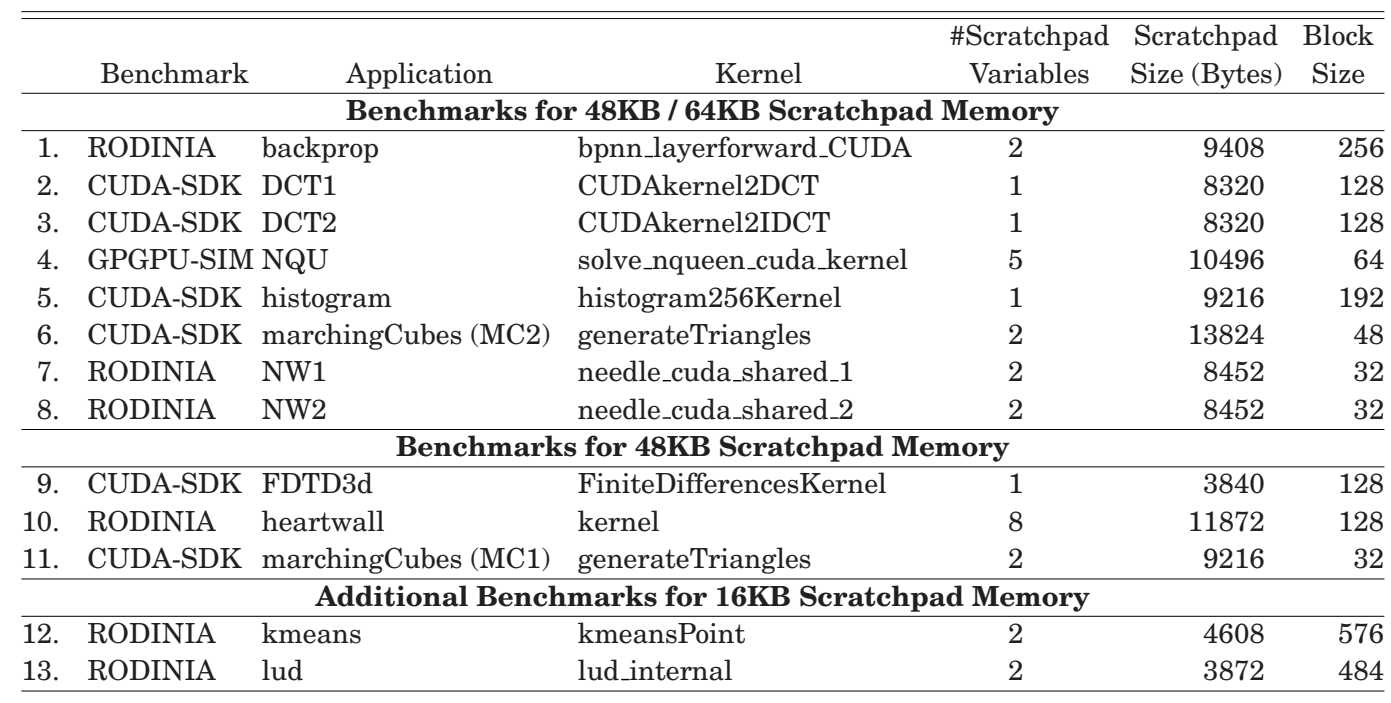

block parameters are similar to that of NVIDIA's Kepler and Maxwell architectures, respectively. The benchmarks that are used for the evaluation are shown in Table VI. The changes in Table VI with respect to those in Table I are:

-Kernel scratchpad memory size for DCT1 and DCT2 is increased from 2112 to 8320. This change ensures that applications are limited by scratchpad memory for both the configurations.

-A new application, $M C 2$, is created based on $M C 1$ - the only difference being that kernel scratchpad memory size is increased to 13824, this is to enable scratchpad sharing for Configuration-2.

- The applications DCT3, DCT4, SRAD1, and SRAD2 are dropped as scratchpad sharing could not be made applicable even by increasing the kernel scratchpad memory size. These applications are limited either by the number of thread blocks or by the number of threads.

Note that scratchpad sharing is applicable for MC1, FDTD3d, and heartwall only with Configuration-1, but not with Configuration-2 (Table VII). In addition, we performed experiments with $16 \mathrm{~KB}$ scratchpad memory per SM for two new applications, kmeans and lud. 
Table VII. Other GPGPU-Sim Configurations

\begin{tabular}{l|r|r}
\hline \hline \multirow{2}{*}{ Resource/Core } & \multicolumn{2}{|c}{ Configuration } \\
\cline { 2 - 3 } Scratchpad Memory & $48 \mathrm{~KB}$ & \#2 \\
Max Thread Blocks & 16 & 32 \\
Max Threads & 2048 & 2048 \\
Number of SMs & 14 & 14 \\
\hline
\end{tabular}

Table VIII. Benchmarks Used for Comparison with Shared Memory Multiplexing [Yang et al. 2012]

\begin{tabular}{llccc}
\hline \hline Application & \multicolumn{1}{c}{ Kernel } & $\begin{array}{c}\text { \#Scratchpad } \\
\text { Variables }\end{array}$ & $\begin{array}{c}\text { Scratchpad } \\
\text { Size (Bytes) }\end{array}$ & $\begin{array}{c}\text { Block } \\
\text { Size }\end{array}$ \\
\hline Matrix Vector Multiplication (MV) & mv_shared & 1 & 4224 & 32 \\
Fast Fourier Transform (FFT) & kfft & 1 & 8704 & 64 \\
MarchingCubes (MC) & generateTriangles & 2 & 9216 & 32 \\
ScalarProd (SP) & scalarProdGPU & 1 & 4114 & 64 \\
Histogram (HG) & histogram256 & 1 & 7168 & 32 \\
Convolution (CV) & convolutionColumnsKernel & 1 & 8256 & 128 \\
\hline
\end{tabular}

Figure 21 shows the performance comparison of our approach with the two baseline configurations in terms of number of instructions executed per cycle. In the figure, we use Unshared-LRR-48K to denote the baseline approach that uses $48 \mathrm{~KB}$ scratchpad memory (according to Configuration-1) and Shared-OWF-OPT-48K to denote the scratchpad sharing approach (with all optimizations) that use $48 \mathrm{~KB}$ scratchpad memory. The notations Unshared-LRR-64K and Shared-OWF-OPT-64K, which use 64KB scratchpad memory configuration, are defined analogously.

We observe that with Shared-OWF-OPT-48K, all the applications except FDTD3d and DCT2 show performance improvement when compared to Unshared-LRR-48K. Similarly with Shared-OWF-OPT-64K, all the applications perform better when compared to Unshared-LRR-64K. Consider the applications backprop, DCT1, histogram, $M C 2$, and $N Q U$. These applications with Shared-OWF-OPT-48K perform better even when compared to Unshared-LRR-64K. Also, these applications, when used with Shared-OWF-OPT-64K, perform better than Unshared-LRR-64K as well. NW1 and NW2 show improvement with scratchpad sharing when compared to their respective baseline approaches. Applications heartwall and MC1, where scratchpad sharing is applicable only with Configuration-1 (Table VII), show improvement when compared to Unshared-LRR-48K. For DCT2, increasing the amount of scratchpad memory from $48 \mathrm{~KB}$ to $64 \mathrm{~KB}$ per SM does not improve the performance of Unshared-LRR configuration, since it increases number of stall cycles in the SM. Hence increase in the number of thread blocks for Shared-OWF-OPT-48K does not improve its performance w.r.t Unshared-LRR-48K. However, Shared-OWF-OPT-48K performs better than SharedOWF-OPT-64K, because Shared-OWF-OPT-48K has lesser number of thread blocks that make more progress, hence reducing resource contention. FDTD3d does not show improvement with scratchpad sharing due to increase in the stall cycles with our approach. Also additional benchmarks, kmeans and lud show improvement with SharedOWF-OPT-16K when compared to Unshared-LRR-16K. To summarize, our approach helps in improving the performance of the applications even with increase in the size of the scratchpad memory per SM.

7.3.2. Performance Comparison with Shared Memory Multiplexing [Yang et al. 2012]. Figure 22 compares the performance of our approach with the software approaches proposed by Yang et al. [2012] in terms of number of simulation cycles. We use their benchmarks (Table VIII), and simulate them on the GPU configuration shown in Table II. In 


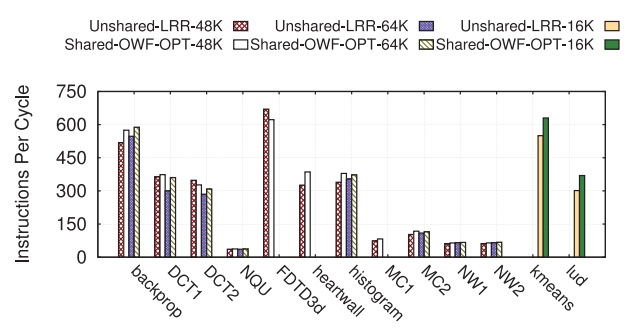

Fig. 21. Performance analysis for various configurations.

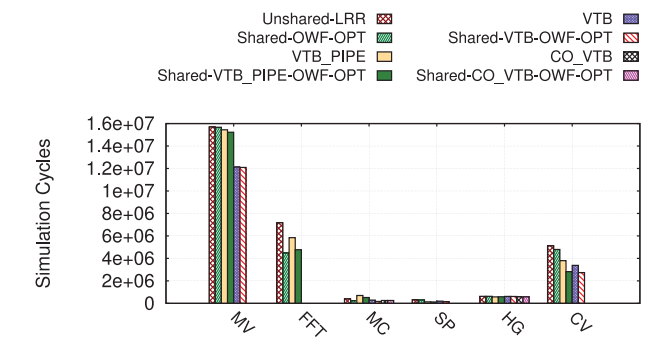

Fig. 22. Performance comparison with other approaches (lower value is better).

the figure, Unshared-LRR and Shared-OWF-OPT denote the baseline and scratchpad sharing approaches, respectively. VTB, VTB_PIPE, and CO_VTB denote the compiler optimizations proposed by Yang et al. [2012]. ${ }^{8}$ Similarly, we use Shared-VTB-OWFOPT, Shared-VTB_PIPE-OWF-OPT, and Shared-CO_VTB-OWF-OPT to measure performance of scratchpad sharing on the applications that are optimized with VTB, $V T B \_P I P E$, and $C O \_V T B$, respectively.

In the experiments, we observe a change in the number of executed instructions for $V T B, V T B \_P I P E$, and $C O \_V T B$ approaches, because they require modifications to the benchmarks. The difference in the number of instructions along with their IPC values are reported in Jatala et al. [2016b]. In Figure 22, application $M C$ performs better (spends fewer simulation cycles) with Shared-OWF-OPT than with Unshared$L L R, V T B, V T B \_P I P E$, and $C O \_V T B$ approaches. Interestingly, applying Shared-OWF$O P T$ on top of $V T B, V T B \_P I P E$, or $C O \_V T B$ improves the performance further. Similarly, $F F T$ shows improvement with Shared-OWF-OPT when compared to Unshared$L R R$ and VTB_PIPE. In this case also Shared-VTB_PIPE-OWF-OPT outperforms VTB_PIPE. In contrast, for the application $H G$, sharing does not impact the performance, even on the top of VTB and VTB_PIPE optimizations. This is because the additional thread blocks launched do not make much progress before they start accessing shared scratchpad. For the same reason, scratchpad sharing does not impact on $M V$. The applications $C V$ and SP perform better with VTB_PIPE than with Shared$O W F-O P T$. However, the performance is further improved when scratchpad sharing is combined with VTB and VTB_PIPE approaches. It can be concluded from these experiments that scratchpad sharing and shared memory multiplexing approaches compliment each other well, and most applications show the best performance when the two approaches are combined.

\section{RELATED WORK}

Resource sharing technique [Jatala et al. 2016a], proposed by the authors earlier, improves the throughput by minimizing the register and scratchpad memory underutilization by modifying the GPU architecture and scheduling algorithm. They further propose two optimizations, viz. Unrolling and Reordering of Register Declarations and Dynamic Warp Execution, to improve register utilization and reduce long latencies of register sharing. This work improves their approach by introducing compiler optimizations for better layout of scratchpad variables. It also proposes an algorithm to

\footnotetext{
${ }^{8}$ Note that, as described in Yang et al. [2012], $C O \_V T B$ is suitable only for few workloads (i.e., $M C$ and $H G$ ). Also, for FFT, we do not compare VTB with VTB_PIPE, because VTB combines four thread blocks, whereas $V T B \_P I P E$ combines two thread blocks in their implementation.
} 
promote early release of shared scratchpad with the help of new relssp instructions. Other related approaches that improve the performance of GPUs are discussed below:

Resource Management in GPUs. Shared memory multiplexing [Yang et al. 2012] proposes solutions that come closest to our approach. They provide software and hardware solutions to address the TLP problem caused by limited shared memory. The software approach combines two thread blocks into a single virtual thread block. The two thread blocks in a virtual block can execute instructions in parallel, as long as they do not access shared memory; and become serial when they need to access shared memory. The article also describes a mechanism (called CO-VTB) that divides the shared memory into private and public part so the thread blocks in a virtual block can access the private part in parallel and the public part in serial. However, CO-VTB has a high overhead of partitioning the data into private and public part, and is not suitable for all workloads. Also, they need to generate the code manually. The article also gives a hardware solution to dynamically allocate and deallocate scratchpad memory using the existing barrier instruction. Again, these instructions need to be inserted manually in the code, and nesting of the barrier instructions is not allowed to avoid any deadlocks.

In contrast, ours is a hardware solution that allows launching additional thread blocks in each SM. These additional thread blocks use the wasted scratchpad memory and also share part of the allocated scratchpad memory with other resident thread blocks. The additional thread blocks launched in our approach make progress as long as they do not require shared scratchpad memory, and wait until the shared scratchpad is released by other thread blocks. Our approach is fully automatic-our compiler optimization automatically identifies the regions of the shared and unshared scratchpad memory and inserts instruction to release the shared scratchpad as early as possible. Even in the presence of barrier instructions, our approach cannot have deadlocks. In addition, we propose a warp scheduling mechanism that effectively schedule these additional warps to hide the long latencies in a better way. Section 7.3.2 compares our work with theirs quantitatively, on the same benchmarks.

Warp level divergence technique [Xiang et al. 2014] improves the TLP by minimizing register underutilization. It launches one additional partial thread block when there are insufficient number of registers for an entire thread block. However, the number of warps in the partial thread block is decided by the number of unutilized registers, and also the partial thread block does not share registers with any other thread blocks. The unified storage approach [Gebhart et al. 2012] allocates the resources of SM (such as registers, scratchpad memory, and cache) dynamically as per the application demand. Tarjan and Skadron [2011] use virtual registers to launch more thread blocks. These registers are mapped to the physical registers as per the demand. Our compiler optimizations can help in early release of unused registers with this approach. Gomez-Luna et al. [2013] describe a mechanism to lock and unlock parts of scratchpad memory. We can reutilize the existing mechanism by defining a custom hash function that maps shared scratchpad memory regions to corresponding lock addresses. For unshared scratch region, the access can be given directly. Kayiran et al. [2013] propose a dynamic algorithm to launch the optimal number of thread blocks in an SM to reduce the resource contention. We can combine their techniques with our approach to reduce the increase in the stall cycles that occur with shared thread blocks. Li et al. [2011] propose a resource virtualization scheme for sharing of GPU resources with multiprocessors. The virtualization layer proposed by them helps in improving the performance by overlapping multiple kernels executions.

Compiler Optimizations for Efficient Resource Utilization in GPUs. Ma and Agrawal [2010] formulated the problem of scratchpad memory allocation as an integer 
programming problem, which maximizes scratchpad memory access and minimizes device memory access to improve GPU performance. Their framework can allocate parts of arrays on scratchpad, and also suggest profitable loop transformations. Hayes and Zhang [2014] proposed on-chip memory allocation scheme for efficient utilization of GPU resources. It aims to alleviate register pressure by spilling registers to scratchpad memory instead of local memory. Xie et al. [2015] proposed a compile time coordinated register allocation scheme to minimize the cost of spilling registers. These schemes do not propose any architectural change to GPUs and are orthogonal to our approach of scratchpad sharing.

Scheduling Techniques for GPUs. The two-level warp scheduling algorithm, proposed by Narasiman et al. [2011], forms groups of warps and uses LRR to schedule warps in a group. It also proposes a large warp microarchitecture to minimize resource underutilization. Lee and $\mathrm{Wu}$ [2014] hide the long execution latencies by scheduling critical warps more frequently than other than warps. It helps in finishing the thread block sooner thus improving resource utilization. However, it requires the knowledge of critical warps. To address the problem, Lee et al. [2015] proposed a coordinated solution that identifies the critical warps at run-time using instructions and stall cycles. Further, they proposed a greedy based critical warp scheduling algorithm to accelerate the critical warps in the SMs. OWL [Jog et al. 2013] provides a scheduling mechanism to reduce cache contention and to improve DRAM bank level parallelism. Lee et al. [2014] focus on reducing resource contention by providing lazy thread block scheduling mechanism. They also proposed block level CTA scheduling policy that allocates consecutive CTAs into the same SM to exploit cache locality.

Improving GPU Performance through Memory Management. Several other approaches exploit memory hierarchy to improve the performance of GPU applications. $\mathrm{Li}$ et al. [2015b] proposed compiler techniques to efficiently place data onto registers, scratchpad memory, and global memory by analyzing data access patterns. Sethia et al. [2015] proposed a scheduling policy that improves the GPU performance of memory intensive workloads. Their approach detects memory saturation events and prioritizes the memory requests of a single warp to improve cache hit rate. Li et al. [2015a] provide a mechanism to handle the cache contention problem that occurs due to increased number of resident threads in an SM. Their approach is alternative to the thread throttling techniques [Rogers et al. 2012; Kayiran et al. 2013].

Problems with Warp Divergence. Other techniques to improve GPU performance is by handling warp divergence. Dynamic warp formation [Fung et al. 2007] addresses the limited thread level parallelism that is present due to branch divergence. It dynamically forms new warps based on branch target condition. However, the performance of this approach is limited by the warp scheduling policy. Thread block compaction [Fung and Aamodt 2011] addresses the limitation of dynamic warp formation that occurs when the new warps that are formed may require more number of memory accesses. Their approach provides a solution by regrouping the new warps at the reconverging points. However, in their solution, warps need to wait for other warps to reach the divergent path. Anantpur and Govindarajan [2014] proposed linearization technique to avoid duplicate execution of instructions that occurs due to branch divergence in GPUs. Brunie et al. [2012] and Han and Abdelrahman [2011] provide hardware and software solutions to handle branch divergence in GPUs.

Miscellaneous. Warped pre-execution [Lee et al. 2016] accelerates a single warp by executing independent instructions when a warp is stalled due to long latency 
instruction. Huo et al. [2010] and Gutierrez et al. [2008] show that several applications are improved by using scratchpad memory instead of using global memory.

\section{CONCLUSIONS AND FUTURE WORK}

In this article, we propose architectural changes and compiler optimizations for sharing scratchpad effectively to address the underutilization of scratchpad memory in GPUs. Experiments with various benchmarks help us conclude that if the number of resident thread blocks launched by an application is limited by scratchpad (Table I), scratchpad sharing (with the compiler optimizations) improves the performance. On the other hand, for other applications where the number of thread blocks is not limited by scratchpad (Table IV), the hardware changes do not negatively impact the run-time.

We observe that scratchpad sharing will be more effective if programs that need scratchpad memory start accessing it as late as possible and completes as early as possible. This allows additional thread blocks to increase the TLP by executing more number of instructions before they start accessing shared scratchpad memory.

In future, we would like to extend our work to integrate register sharing approach [Jatala et al. 2016a]. Value range analysis techniques [Harrison 1977; Quintao Pereira et al. 2013], typically employed for detecting buffer overflows, can be incorporated in our approach to refine the access ranges of shared scratchpad variables, thus help release shared scratchpad even earlier. We need to study the impact of hardware changes on power consumption and find ways to minimize it.

\section{ACKNOWLEDGMENTS}

We thank the anonymous reviewers for their useful feedback. We also thank the authors of Yang et al. [2012] for sharing their benchmarks with us.

\section{REFERENCES}

Jayvant Anantpur and R. Govindarajan. 2014. Taming control divergence in GPUs through control flow linearization. In Proceedings of the Conference on Compiler Construction (CC'14).

A. Bakhoda, G. L. Yuan, W. W. L. Fung, H. Wong, and T. M. Aamodt. 2009. Analyzing CUDA workloads using a detailed GPU simulator. In Proceedings of the IEEE International Symposium on Performance Analysis of Systems and Software.

Nicolas Brunie, Sylvain Collange, and Gregory Diamos. 2012. Simultaneous branch and warp interweaving for sustained GPU performance. In Proceedings of the International Symposium on Computer Architecture.

Shuai Che, M. Boyer, Jiayuan Meng, D. Tarjan, J. W. Sheaffer, Sang-Ha Lee, and K. Skadron. 2009. Rodinia: A benchmark suite for heterogeneous computing. In Proceedings of the IEEE International Symposium on Workload Characterization.

CUDA 2012. CUDA C Programming Guide. (2012). Retrieved from http://docs.nvidia.com/cuda/pdf/CUDA C_Programming_Guide.pdf.

CUDA-SDK 2014. CUDA-SDK. (2014). Retrieved from http://docs.nvidia.com/cuda/cuda-samples.

Gregory Frederick Diamos, Andrew Robert Kerr, Sudhakar Yalamanchili, and Nathan Clark. 2010. Ocelot: A dynamic optimization framework for bulk-synchronous applications in heterogeneous systems. In Proceedings of the Conference on Parallel Architectures and Compilation Techniques.

Wilson W. L. Fung and Tor M. Aamodt. 2011. Thread block compaction for efficient SIMT control flow. In Proceedings of the Conference on High Performance Computer Architecture.

Wilson W. L. Fung, Ivan Sham, George Yuan, and Tor M. Aamodt. 2007. Dynamic warp formation and scheduling for efficient GPU control flow. In Proceedings of the International Symposium on Microarchitecture.

Mark Gebhart, Stephen W. Keckler, Brucek Khailany, Ronny Krashinsky, and William J. Dally. 2012. Unifying primary cache, scratch, and register file memories in a throughput processor. In Proceedings of the International Symposium on Microarchitecture. 
Juan Gomez-Luna, Jose Maria Gonzalez-Linares, Jose Ignacio Benavides Benitez, and Nicolas Guil. 2013. Performance modeling of atomic additions on GPU scratchpad memory. IEEE Trans. Parallel Distrib. Syst. (2013).

GPGPUSIM 2014. GPGPU-Sim Simulator. (2014). Retrieved from http://www.gpgpu-sim.org.

Eladio Gutierrez, Sergio Romero, Maria A. Trenas, and Emilio L. Zapata. 2008. Memory locality exploitation strategies for FFT on the CUDA architecture. In Proceedings of the International Meeting on HighPerformance Computing for Computational Science.

Tianyi David Han and Tarek S. Abdelrahman. 2011. Reducing branch divergence in GPU programs. In Proceedings of the Workshop on General Purpose Processing on Graphics Processing Units.

William H. Harrison. 1977. Compiler analysis of the value ranges for variables. IEEE Trans. Software Eng. 3 , 3 (1977).

Ari B. Hayes and Eddy Z. Zhang. 2014. Unified on-chip memory allocation for SIMT architecture. In Proceedings of the International Conference on Supercomputing.

Xin Huo, V. T. Ravi, Wenjing Ma, and G. Agrawal. 2010. Approaches for parallelizing reductions on modern GPUs. In Proceedings of the Conference on High Performance Computing.

Vishwesh Jatala, Jayvant Anantpur, and Amey Karkare. 2015. The more we share, the more we have: Improving GPU performance through register sharing. CoRR abs/1503.05694 (2015).

Vishwesh Jatala, Jayvant Anantpur, and Amey Karkare. 2016a. Improving GPU performance through resource sharing. In Proceedings of the Conference on High-Performance Parallel and Distributed Computing (HPDC'16).

Vishwesh Jatala, Jayvant Anantpur, and Amey Karkare. 2016b. Scratchpad sharing in GPUs. CoRR abs/1607.03238 (2016).

Adwait Jog, Onur Kayiran, Nachiappan Chidambaram Nachiappan, Asit K. Mishra, Mahmut T. Kandemir, Onur Mutlu, Ravishankar Iyer, and Chita R. Das. 2013. OWL: Cooperative thread array aware scheduling techniques for improving GPGPU performance. In Proceedings of the Conference on Architectural Support for Programming Languages and Operating Systems.

John B. Kam and Jeffrey D. Ullman. 1976. Global data flow analysis and iterative algorithms. J. ACM 23, 1 (Jan. 1976).

O. Kayiran, A. Jog, M. T. Kandemir, and C. R. Das. 2013. Neither more nor less: Optimizing thread-level parallelism for GPGPUs. In Proceedings of the Conference on Parallel Architectures and Compilation Techniques.

Uday Khedker, Amitabha Sanyal, and Bageshri Karkare. 2009. Data Flow Analysis: Theory and Practice (1st ed.). CRC Press, Inc., Boca Raton, FL.

Minseok Lee, Seokwoo Song, Joosik Moon, J. Kim, Woong Seo, Yeongon Cho, and Soojung Ryu. 2014. Improving GPGPU resource utilization through alternative thread block scheduling. In Proceedings of the Conference on High Performance Computer Architecture.

Sangpil Lee, Won Woo Ro, Keunsoo Kim, Gunjae Koo, Myung Kuk Yoon, and Murali Annavaram. 2016. Warped-preexecution: A GPU pre-execution approach for improving latency hiding. In Proceedings of the Conference on High Performance Computer Architecture.

Shin-Ying Lee, Akhil Arunkumar, and Carole-Jean Wu. 2015. CAWA: Coordinated warp scheduling and cache prioritization for critical warp acceleration of GPGPU workloads. In Proceedings of the International Symposium on Computer Architecture.

Shin-Ying Lee and Carole-Jean Wu. 2014. CAWS: Criticality-aware warp scheduling for GPGPU workloads. In Proceedings of the Conference on Parallel Architectures and Compilation Techniques.

Chao Li, Yi Yang, Zhen Lin, and Huiyang Zhou. 2015b. Automatic data placement into GPU on-chip memory resources. In Proceedings of the Conference on Code Generation and Optimization.

Dong Li, Minsoo Rhu, Daniel R. Johnson, Mike O’Connor, Mattan Erez, Doug Burger, Donald S. Fussell, and Stephen W. Redder. 2015a. Priority-based cache allocation in throughput processors. In Proceedings of the Conference on High Performance Computer Architecture.

T. Li, V. K. Narayana, E. El-Araby, and T. El-Ghazawi. 2011. GPU resource sharing and virtualization on high performance computing systems. In Proceedings of the International Conference on Parallel Processing.

Wenjing Ma and Gagan Agrawal. 2010. An integer programming framework for optimizing shared memory use on GPUs. In Proceedings of the Conference on Parallel Architectures and Compilation Techniques.

Veynu Narasiman, Michael Shebanow, Chang Joo Lee, Rustam Miftakhutdinov, Onur Mutlu, and Yale N. Patt. 2011. Improving GPU performance via large warps and two-level warp scheduling. In Proceedings of the International Symposium on Microarchitecture.

OpenCL 2009. Retrieved from https://www.khronos.org/opencl/ Accessed 2012. 
PTX 2014. Parallel Thread Execution. (2014). Retrieved from http://docs.nvidia.com/cuda/parallel-threadexecution/.

Fernando Magno Quintao Pereira, Raphael Ernani Rodrigues, and Victor Hugo Sperle Campos. 2013. A fast and low-overhead technique to secure programs against integer overflows. In Proceedings of the Conference on Code Generation and Optimization.

Timothy G. Rogers, Mike O'Connor, and Tor M. Aamodt. 2012. Cache-conscious wavefront scheduling. In Proceedings of the International Symposium on Microarchitecture.

Ankit Sethia, D. Anoushe Jamshidi, and Scott Mahlke. 2015. Mascar: Speeding up GPU warps by reducing memory pitstops. In Proceedings of the Conference on High Performance Computer Architecture.

D. Tarjan and K. Skadron. 2011. On demand register allocation and deallocation for a multithreaded processor. Retrieved from http://www.google.com/patents/US20110161616. (2011). U.S. Patent App. 12/649,238.

Ping Xiang, Yi Yang, and Huiyang Zhou. 2014. Warp-level divergence in GPUs: Characterization, impact, and mitigation. In Proceedings of the Conference on High Performance Computer Architecture.

Xiaolong Xie, Yun Liang, Xiuhong Li, Yudong Wu, Guangyu Sun, Tao Wang, and Dongrui Fan. 2015. Enabling coordinated register allocation and thread-level parallelism optimization for GPUs. In Proceedings of the International Symposium on Microarchitecture.

Yi Yang, Ping Xiang, Mike Mantor, Norm Rubin, and Huiyang Zhou. 2012. Shared memory multiplexing: A novel way to improve GPGPU throughput. In Proceedings of the Conference on Parallel Architectures and Compilation Techniques.

Received July 2016; revised February 2017; accepted March 2017 